\title{
A novel scenario in the semi-constrained NMSSM
}

\author{
Kun Wang and Jingya Zhu ${ }^{1}$ \\ Center for Theoretical Physics, School of Physics and Technology, \\ Wuhan University, Wuhan 430072, China \\ E-mail: wk2016@whu.edu.cn, zhujy@whu.edu.cn
}

ABSTRACT: In this work, we develop a novel efficient scan method, combining the Heuristically Search (HS) and the Generative Adversarial Network (GAN), where the HS can shift marginal samples to perfect samples, and the GAN can generate a huge amount of recommended samples from noise in a short time. With this efficient method, we find a new scenario in the semi-constrained Next-to Minimal Supersymmetric Standard Model (scNMSSM), or NMSSM with non-universal Higgs masses. In this scenario, (i) Both muon g-2 and right relic density can be satisfied, along with the high mass bound of gluino, etc. As far as we know, that had not been realized in the scNMSSM before this work. (ii) With the right relic density, the lightest neutralinos are singlino-dominated, and can be as light as $0-12 \mathrm{GeV}$. (iii) The future direct detections XENONnT and LUX-ZEPLIN (LZ-7 2T) can give strong constraints to this scenario. (iv) The current indirect constraints to Higgs invisible decay $h_{2} \rightarrow \tilde{\chi}_{1}^{0} \tilde{\chi}_{1}^{0}$ are weak, but the direct detection of Higgs invisible decay at the future HL-LHC may cover half of the samples, and that of the CEPC may cover most. (v) The branching ratio of Higgs exotic decay $h_{2} \rightarrow h_{1} h_{1}, a_{1} a_{1}$ can be over 20 percent, while their contributions $\left(h_{2} \rightarrow 4 \tilde{\chi}_{1}^{0}\right)$ to the invisible decay are very small.

KEYWoRds: Supersymmetry Phenomenology

ARXIV EPRINT: 2002.05554

\footnotetext{
${ }^{1}$ Corresponding author.
} 


\section{Contents}

1 Introduction 1

2 The semi-constrained NMSSM and the search strategy 2

2.1 The Higgs and electroweakinos sector of the scNMSSM 3

2.2 The Heuristically Search (HS) 5

2.3 The Generative Adversarial Network (GAN) 6

3 Results and discussions $\quad 8$

3.1 Scan with HS and GAN 8

$\begin{array}{ll}3.2 & \text { Light dark matter (DM) and Higgs invisible decay } \\ \end{array}$

$\begin{array}{llr}4 \text { Conclusions } & 18\end{array}$

\section{Introduction}

Higgs boson was discovered in 2012 [1, 2], and its production rate in most channels coincides with the Standard Model (SM) prediction considering uncertainties [3-5]. While there are still chances for physics beyond the SM. For example, for the branching ratio of Higgs boson invisible decay, the current excluding limits are only $26 \%$ by ATLAS [6] and $19 \%$ by CMS [7], with all data at Run I and data of about $36 \mathrm{fb}^{-1}$ at Run II.

Supersymmetry is a popular theory beyond the SM, which introduces a new internal symmetry between fermions and bosons. Thus the large hierarchy problem can be solved, gauge coupling can be unified, and dark matter (DM) candidates can be provided, etc. In the Minimal Supersymmetric Standard Model (MSSM) with 7 free parameters at the electroweak scale, a SM-like $125 \mathrm{GeV}$ Higgs can be afforded, but need large fine-tuning, and the branching ratio of Higgs boson invisible decay can be about $10 \%$ at most [810]. The Next-to Minimal Supersymmetric Standard Model (NMSSM) with $\mathbb{Z}_{3}$ symmetry extends the MSSM by a complex singlet superfield $\hat{S}$, but introduces four more parameters. In the graceful and simple model of fully-constrained NMSSM (cNMSSM), all Higgs and sfermion masses are assumed to be unified at the Grand Unified theoretical (GUT) scale, thus only four parameters at GUT scale are left free [11-19]. These four or five parameters run according to the Renormalization Group Equations (RGEs), forming the spectrum of NMSSM at low energy scale. While it was found that when considering all the constraints including muon g-2, the SM-like Higgs mass can not reach to $125 \mathrm{GeV}$ in the $\operatorname{cNMSSM}^{1}[11$, 12], like these in the CMSSM, NUHM1 and NUHM2 [21-24].

\footnotetext{
${ }^{1}$ Notice that there are also some other ways to solve this problem, e.g., introducing right-handed neutrinos to the cNMSSM [20].
} 
In this work, we consider possible scenarios of Higgs invisible decay in the semiconstrained NMSSM (scNMSSM) [25-30], which relaxing the Higgs masses at the GUT scale, and also called NMSSM with non-universal Higgs mass (NUHM). As a simple and graceful SUSY model, the scNMSSM had attracted much attention. In refs. [27, 28] the constraints of LHC and dark matter to scNMSSM was considered, while the muon g-2 was left aside; in ref. [26] muon g-2 was satisfied, while dark matter relic density is not sufficient; in refs. [25, 29, 30], direct searches for the higgsino sector was considered; in ref. [31], the extended model with right-handed neutrinos was considered. In this work, we consider all constraints including muon g-2, and also try to get sufficient relic density.

In this work, to include constraints of muon g-2, etc., get sufficient relic density, and get as-large-as-possible branching ratio of Higgs invisible decay, we developed a novel efficient method to scan the parameter space, which consists of the Heuristically Search (HS) and the Generative Adversarial Network (GAN). Note that in refs. [32, 33], the Machine Learning (ML) scan method has been used to explore the parameter space, and a scanning tool xBIT [34] based on ML has been developed. This ML scan is based on several classifiers, dealing with a Classification problem that each sample gets a probability of how much it could be a perfect sample. This scan method also needs to generate samples in highdimension space, which will cost very long time, (eg., when the dimension is 9 and each dimension has 100 grid, at least $100^{9}$ samples need to be generated). On the contrary, we adopt a generative model, the Generative Adversarial Network (GAN) [35], which is a famous star in deep learning area and also gets much attention in high energy physics [3644]. The GAN can directly generate samples with the similar distribution as the training samples. So with a well-trained GAN, we can get as many recommended samples as we want. And with the HS we developed, we have a chance to shift some 'bad' or 'marginal' samples to 'good' samples. Combined with HS and GAN, we developed a novel method that can get a huge amount of surviving samples in a short time. Then, we used this novel efficient method to study the parameter space of scNMSSM, under current constraints including LHC, B physics, muon g-2, and dark matter, etc. We require our surviving samples to satisfy all these constraints, and part of them predict right relic density. To study Higgs invisible decay, we require the LSP mass lighter than half of the SM-like Higgs mass $\left(m_{\tilde{\chi}_{1}^{0}}<m_{h_{\mathrm{SM}}} / 2\right)$, and the invisible branching ratio be as large as possible. As can be seen from the following sections, this method is powerful in getting this novel scenario in the scNMSSM.

The rest of this paper is organized as follows. In section 2, we briefly introduce the Higgs and electroweakino sectors of the scNMSSM, and our search strategy consisting of HS and GAN. In section 3, we describe the detail of our scan process and then discuss the Higgs invisible decay and light dark matter in the scNMSSM. Finally, we draw our conclusions in section 4 .

\section{The semi-constrained NMSSM and the search strategy}

The NMSSM extends the MSSM particle content by adding a singlet superfield $\hat{S}$, which provides an effective $\mu$-term. The superpotential of the $\mathbb{Z}_{3}$-invariant NMSSM is

$$
W_{\mathrm{NMSSM}}=y_{u} \hat{Q} \cdot \hat{H}_{u} \hat{u}^{c}+y_{d} \hat{Q} \cdot \hat{H}_{d} \hat{d}^{c}+y_{u} \hat{L} \cdot \hat{H}_{d} \hat{e}^{c}+\lambda \hat{S} \hat{H}_{u} \cdot \hat{H}_{d}+\frac{\kappa}{3} \hat{S}^{3}
$$


where the hats are used for superfields, $y_{u, d, e}$ stand for corresponding Yukawa couplings, and $\lambda, \kappa$ are dimensionless coupling constants. When the singlet superfield $\hat{S}$ gets a vacuum expectation value $(\mathrm{VEV}),\langle S\rangle=v_{s}$, a effective $\mu$-term is generated dynamically from the term $\lambda \hat{S} \hat{H}_{u} \cdot \hat{H}_{d}$, with

$$
\mu_{\mathrm{eff}}=\lambda v_{s} .
$$

For convenience, in the following we refer to $\mu_{\text {eff }}$ as $\mu$. And the VEVs of the two doublet Higgs superfields $\hat{H}_{u}$ and $\hat{H}_{d}$ are $v_{u}$ and $v_{d}$ respectively, where $v_{u}^{2}+v_{d}^{2}=v^{2}=(174 \mathrm{GeV})^{2}$.

The soft SUSY breaking terms in the NMSSM are only different from the MSSM in several terms:

$$
-\mathcal{L}_{\mathrm{NMSSM}}^{\mathrm{soft}}=-\left.\mathcal{L}_{\mathrm{MSSM}}^{\mathrm{soft}}\right|_{\mu=0}+m_{S}^{2}|S|^{2}+\lambda A_{\lambda} S H_{u} \cdot H_{d}+\frac{1}{3} \kappa A_{\kappa} S^{3}+\text { h.c. },
$$

where the $S, H_{u}$ and $H_{d}$ are the scalar components of the superfields respectively, the $m_{S}^{2}$ is the soft SUSY breaking mass for single field $S$, and the trilinear coupling constants $A_{\lambda}$ and $A_{\kappa}$ have mass dimension.

Unlike that in the CNMSSM or CMSSM, in the scNMSSM the Higgs sector is assumed to be non-universal at the GUT scale. Then, at the GUT scale, the Higgs soft mass $m_{H_{u}}^{2}, m_{H_{d}}^{2}$ and $m_{S}^{2}$ are allowed to be different from $M_{0}^{2}+\mu^{2}$, and the trilinear couplings $A_{\lambda}, A_{\kappa}$ can be different from $A_{0}$. Hence, in the scNMSSM, the complete parameter sector can be usually chosen as

$$
\lambda, \kappa, \tan \beta=\frac{v_{u}}{v_{d}}, \mu, A_{\lambda}, A_{\kappa}, A_{0}, M_{1 / 2}, M_{0}
$$

at the GUT scale. While the parameters at low energy scale can be calculated in the RGEs running from these GUT-scale parameters.

\subsection{The Higgs and electroweakinos sector of the scNMSSM}

When the electroweak symmetry broken, the scalar component of superfields $\hat{H}_{u}, \hat{H}_{d}$ and $\hat{S}$ can be written as

$$
H_{u}=\left(\begin{array}{c}
H_{u}^{+} \\
v_{u}+\frac{H_{u}^{R}+i H_{u}^{I}}{\sqrt{2}}
\end{array}\right), \quad H_{d}=\left(\begin{array}{c}
v_{d}+\frac{H_{d}^{R}+i H_{d}^{I}}{\sqrt{2}} \\
H_{d}^{-}
\end{array}\right), \quad S=v_{s}+\frac{S^{R}+i S^{I}}{\sqrt{2}},
$$

where $H_{u}^{R}, H_{d}^{R}$, and $S^{R}$ are CP-even component fields, $H_{u}^{I}, H_{u}^{I}$, and $S^{I}$ are the CP-odd component fields, and the $H_{u}^{+}$and $H_{d}^{-}$are charged component fields. In practice, it is convenient to rotate the fields as

$$
\begin{aligned}
& H_{1}=\cos \beta H_{u}+\varepsilon \sin \beta H_{d}^{*}=\left(\begin{array}{c}
H^{+} \\
\frac{S_{1}+i P_{1}}{\sqrt{2}}
\end{array}\right) \\
& H_{2}=\sin \beta H_{u}-\varepsilon \cos \beta H_{d}^{*}=\left(\begin{array}{c}
G^{+} \\
v+\frac{S_{2}+i G^{0}}{\sqrt{2}}
\end{array}\right) \\
& H_{3}=S=v_{s}+\frac{S_{3}+i P_{2}}{\sqrt{2}}
\end{aligned}
$$


where $\varepsilon=\left(\begin{array}{cc}0 & 1 \\ -1 & 0\end{array}\right)$, and $H_{2}, H_{1}$ and $H_{3}$ are the SM Higgs doublet, new doublet and singlet respectively.

In the basis $\left(S_{1}, S_{2}, S_{3}\right)$, the CP-even Higgs boson mass matrix $M_{S}^{2}$ is given by $[45,46]$

$$
\begin{aligned}
& M_{S, 11}^{2}=M_{A}^{2}+\left(m_{Z}^{2}-\lambda^{2} v^{2}\right) \sin ^{2} 2 \beta+\Delta M_{S, 11}^{2}, \\
& M_{S, 22}^{2}=m_{Z}^{2} \cos ^{2} 2 \beta+\lambda^{2} v^{2} \sin ^{2} 2 \beta+\Delta M_{S, 22}^{2}, \\
& M_{S, 12}^{2}=-\frac{1}{2}\left(m_{Z}^{2}-\lambda^{2} v^{2}\right) \sin 4 \beta+\Delta M_{S, 12}^{2}, \\
& M_{S, 33}^{2}=\frac{1}{4} \lambda^{2} v^{2}\left(\frac{M_{A}}{\mu / \sin 2 \beta}\right)^{2}+\kappa v_{s} A_{\kappa}+4\left(\kappa v_{s}\right)^{2}-\frac{1}{2} \lambda \kappa v^{2} \sin 2 \beta, \\
& M_{S, 13}^{2}=-\left(\frac{M_{A}^{2}}{2 \mu / \sin 2 \beta}+\kappa v_{s}\right) \lambda v \cos 2 \beta \\
& M_{S, 23}^{2}=2 \lambda \mu v\left[1-\left(\frac{M_{A}}{2 \mu / \sin 2 \beta}\right)^{2}-\frac{\kappa}{2 \lambda} \sin 2 \beta\right],
\end{aligned}
$$

where $M_{A}$ is the mass scale of new doublet with

$$
M_{A}^{2}=\frac{2 \mu\left(A_{\lambda}+\kappa v_{s}\right)}{\sin 2 \beta},
$$

and $\Delta M_{S, 11}^{2}, \Delta M_{S, 22}^{2}$ and $\Delta M_{S, 12}^{2}$ are the important corrections at loop level. The firstorder contributions by stop loops are given by [46]

$$
\begin{aligned}
& \Delta M_{S, 11}^{2}=\frac{3 v^{2} y_{t}^{4} \sin ^{2} 2 \beta}{32 \pi^{2}}\left[\ln \left(\frac{M_{S}^{2}}{m_{t}^{2}}\right)+\frac{X_{t} Y_{t}}{M_{S}^{2}}\left(1-\frac{X_{t} Y_{t}}{12 M_{S}^{2}}\right)\right], \\
& \Delta M_{S, 22}^{2}=\frac{3 v^{2} y_{t}^{4} \sin ^{4} \beta}{8 \pi^{2}}\left[\ln \left(\frac{M_{S}^{2}}{m_{t}^{2}}\right)+\frac{X_{t}^{2}}{M_{S}^{2}}\left(1-\frac{X_{t}^{2}}{12 M_{S}^{2}}\right)\right], \\
& \Delta M_{S, 12}^{2}=\frac{3 v^{2} y_{t}^{4} \sin ^{2} \beta \sin 2 \beta}{16 \pi^{2}}\left[\ln \left(\frac{M_{S}^{2}}{m_{t}^{2}}\right)+\frac{X_{t}\left(X_{t}+Y_{t}\right)}{2 M_{S}^{2}}-\frac{X_{t}^{3} Y_{t}}{12 M_{S}^{2}}\right],
\end{aligned}
$$

where $X_{t}=A_{t}-\mu / \tan \beta, Y_{t}=A_{t}+\mu \tan \beta, M_{S}=\sqrt{m_{\tilde{t}_{1}} m_{\tilde{t}_{2}}}$ is the geometric average of the two stop masses and $A_{t}$ is the trilinear parameter associated with the Yukawa coupling of top quark $y_{t}=m_{t} / v$. To have the SM-like Higgs at about $125 \mathrm{GeV}$, with $\tan \beta \gg 1$ and $\lambda \ll 1$ the loop correction $\Delta M_{S, 22}$ need to be about $(86 \mathrm{GeV})^{2}$, which means heavy stops $\left(M_{S} \sim 10 \mathrm{TeV}\right)$, or large stop mixing parameter $A_{t}$.

In the basis $\left(P_{1}, P_{2}\right)$, the CP-odd Higgs boson mass matrix $M_{P}^{2}$ is

$$
\begin{aligned}
& M_{P, 11}^{2}=M_{A}^{2}, \\
& M_{P, 12}^{2}=\lambda v\left(A_{\lambda}-2 \kappa v_{s}\right), \\
& M_{P, 22}^{2}=\lambda\left(A_{\lambda}+4 \kappa v_{s}\right) \frac{v_{u} v_{d}}{v_{s}}-3 \kappa v_{s} A_{\kappa} .
\end{aligned}
$$

Three CP-even mass eigenstates $h_{i}(i=1,2,3)$ (ordered in mass) are mixed from $S_{i}(i=1,2,3)$, and two CP-odd mass eigenstates $a_{i}(i=1,2)$ (ordered in mass) are mixed 
from $P_{i}(i=1,2)$. The mixings are given by

$$
\left(\begin{array}{c}
h_{1} \\
h_{2} \\
h_{3}
\end{array}\right)=S_{i j}\left(\begin{array}{c}
S_{1} \\
S_{2} \\
S_{3}
\end{array}\right), \quad\left(\begin{array}{c}
a_{1} \\
a_{2}
\end{array}\right)=P_{i j}\left(\begin{array}{c}
P_{1} \\
P_{2}
\end{array}\right),
$$

where the mixing matrix $S_{i j}$ and $P_{i j}$ can diagonalize the mass matrix $M_{S}^{2}$ and $M_{P}^{2}$ respectively.

The neutralino sector consists of five neutralinos. In the gauge-eigenstate basis $\psi^{0}=$ $\left(\tilde{B}, \tilde{W}^{3}, \tilde{H}_{d}^{0}, \tilde{H}_{u}^{0}, \tilde{S}\right)$, the neutralino mass matrix takes the form [47]

$$
M_{\tilde{\chi}^{0}}=\left(\begin{array}{ccccc}
M_{1} & 0 & -c_{\beta} s_{W} m_{Z} & s_{\beta} s_{W} m_{Z} & 0 \\
0 & M_{2} & c_{\beta} c_{w} m_{z} & -s_{\beta} c_{W} m_{Z} & 0 \\
-c_{\beta} s_{W} m_{Z} & c_{\beta} c_{w} m_{z} & 0 & -\mu & -\lambda v_{d} \\
s_{\beta} s_{W} m_{Z} & -s_{\beta} c_{W} m_{Z} & -\mu & 0 & -\lambda v_{u} \\
0 & 0 & -\lambda v_{d} & -\lambda v_{u} & 2 \kappa v_{s}
\end{array}\right)
$$

where $s_{\beta}=\sin \beta, c_{\beta}=\cos \beta, s_{W}=\sin \theta_{W}, c_{W}=\cos \theta_{W}$. The mass eigenstates are denoted by $\tilde{\chi}_{i}^{0}(i=1,2,3,4,5)$ ordered in mass. Hereinafter $\tilde{\chi}_{1}^{0}$ is identified as the LSP.

Combining with eq. (2.12), eq. (2.21)and eq. (2.23),we get a relation [30, 48]:

$$
M_{\tilde{\chi}^{0}, 55}^{2}=4 \kappa^{2} v_{s}^{2}=M_{S, 33}^{2}+\frac{1}{3} M_{P, 22}^{2}-\frac{4}{3} v_{u} v_{d}\left(\frac{\lambda^{2} A_{\lambda}}{\mu}+\kappa\right) .
$$

If the LSP $\tilde{\chi}_{1}^{0}$ is highly singlino-dominated, $h_{1}$ and $a_{1}$ are singlet-dominated, and with a sizable $\tan \beta$, a not-too-large $A_{\lambda}$, and small $\lambda$ and $\kappa$, this equation can become:

$$
m_{\tilde{\chi}_{1}^{0}}^{2} \approx m_{h_{1}}^{2}+\frac{1}{3} m_{a_{1}}^{2}
$$

\subsection{The Heuristically Search (HS)}

Usually, We divide the samples into 2 categories according to whether or not the samples passed all constraints. A sample that violated several constraints might be not good enough, but there is a chance that we can lead it to become a good sample.

In our case, we first leave aside the dark matter and muon g-2 constraints, only imposing other constraints in the NMSSMTools. A sample that passes other constraints will get a score to evaluate how much it violates the dark matter and muon g-2 constraints, and we call it a 'marginal sample'.

In table 1, we classify the samples into 3 types: the bad, marginal and perfect samples. For marginal and perfect samples, they will get a score to value how much they violate the constraints. And we try to shift these marginal samples to satisfy the dark matter and muon g-2 constraints, becoming perfect samples. The score function is given as:

$$
f(\mathbf{X})=\sum_{i=1}^{N} \max \left[1-\frac{O_{\text {Theor.max }}^{i}}{O_{\text {Exp.min }}^{i}}, 0\right]+\max \left[\frac{O_{\text {Theor.min }}^{i}}{O_{\text {Exp.max }}^{i}}-1,0\right],
$$




\begin{tabular}{|c|ccc|}
\hline & Type 1 & Type 2 & Type 3 \\
\hline $\begin{array}{c}\text { The basic } \\
\text { constraints }\end{array}$ & $\times$ & $\checkmark$ & $\checkmark$ \\
\hline $\begin{array}{c}\text { The dark matter and } \\
\text { muon g-2 constraints }\end{array}$ & - & $\times$ & $\checkmark$ \\
\hline & bad samples & marginal samples & perfect samples \\
\hline Score & None & $>0$ & $=0$ \\
\hline
\end{tabular}

Table 1. The three types of samples: the bad, marginal and perfect samples.

where $\mathbf{X}$ represent a marginal sample, $O^{i}$ means the $i$-th observable depending on $\mathbf{X}$, the $N$ means there are $\mathrm{N}$ kinds of different observables, the $O_{\text {Theor.min }}^{i}$ and $O_{\text {Theor.max }}^{i}$ are calculated with NMSSMTools, and the $O_{\text {Exp.min }}^{i}$ and $O_{\text {Exp.max }}^{i}$ are given by experimental results. When the score is large, it means the marginal sample violates the experiments more; while when the score is zero, it means the marginal sample becomes a perfect sample, and satisfies all constraints very well, including dark matter and muon g-2 constraints.

In algorithm 1, we give the Heuristically Search algorithm, which can shift a marginal sample to a perfect sample satisfying all constraints. With a marginal sample, $\mathbf{X}$, we search around it and try to find another marginal sample with a smaller score. Then we repeat the process, until we meet a perfect sample whose score is zero, or get failed.

The search can be successful or get failed. Most of the time in our case, the Heuristically Search can lead about $80 \%$ (even over 94\%) marginal samples to perfect samples. Meanwhile, to avoid the program being trapped in a local minimum, we give it a chance to give up. During the search, if the search step is larger than the maximum step $N_{\max }$ (we set it to 20), or the number of tries in one step is larger than the maximum number, $T_{\max }$ (we set it to 50), we stop the program and the search gets failed.

To get a new marginal sample $\mathbf{X}^{\prime}$ around the $\mathbf{X}$, we can treat each component $\mathbf{x}_{i}$ $(i=1 \ldots 9)$ independently. The simplest way is choosing samples around the $\mathbf{x}_{i}$ within radius $r_{i}$ with uniform distribution. To improve the efficiency, the Gaussian distribution is adopted, since it has some chance to search samples far away and could jump out of the local minimums. The Gaussian distribution function of $\mathbf{x}_{i}^{\prime}$ is given as:

$$
\begin{aligned}
f\left(\mathbf{x}^{\prime}{ }_{i}\right) & =\frac{1}{\sqrt{2 \pi} \sigma_{i}} \exp \left[-\frac{\left(x_{i}^{\prime}-x_{i}\right)^{2}}{2 \sigma_{i}^{2}}\right], \\
\sigma_{i} & =r_{i}\left(x_{i, \max }-x_{i, \min }\right),
\end{aligned}
$$

where $r_{i}$ (we set it to $1 / 50$ ) is an important parameter and determines the search efficient. Actually, $r_{i}$ can change with the score. When the score is nearly zero, it means that a perfect sample is nearby, and then $r_{i}$ can change to a smaller one and vice versa.

\subsection{The Generative Adversarial Network (GAN)}

The Generative Adversarial Network (GAN) is a Generative model. It can generate samples with similar distribution as the real data. There are two neural networks in GAN. One is the Generator $\mathbf{G}$, which can generate fake samples. While the other is the Discriminator 


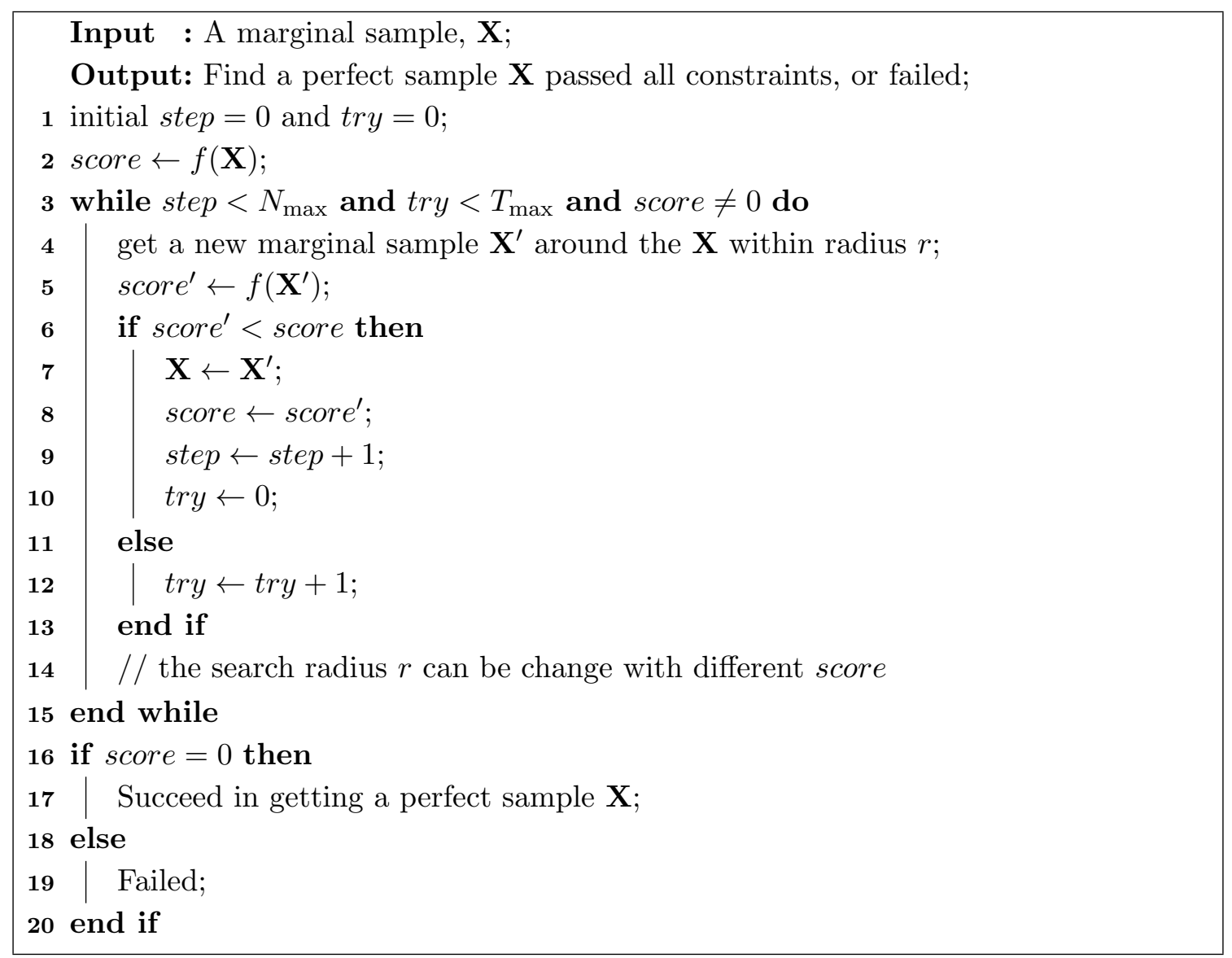

Algorithm 1. The Heuristically Search (HS) with NMSSMTools.

D, which can classify the generated samples into real samples and the fake samples, so it is actually a binary classifier.

When the GAN is being trained, the Discriminator $\mathbf{D}$ tries to classify the generated samples into real and fake samples, meanwhile the Generator $\mathbf{G}$ tries to fool the Discriminator $\mathbf{D}$ and generate almost 'real' samples. After training, the Generator $\mathbf{G}$ and Discriminator $\mathbf{D}$ arrive at a Nash equilibrium. Then we can use the Generator $\mathbf{G}$ to generate 'real' samples as many as we need. And these 'real' samples actually have similar distribution as the real samples coming from the training dataset.

In this work, we use the Artificial Neural Networks to build the Generator $\mathbf{G}$ and the Discriminator D. We adopt a simple Neural Network with 3 hidden layers and each layer with 50 neurons, and the Activation Function is Leaky ReLU. Furthermore, we train our GAN with algorithm 2. In our case, we choose $k=3, n=1, m=20000$, and the training iterations as 2000, while for the Gradient descent we use Adadelta [49].

During the training, we require the Generator to learn the general distribution of the real data, but not try hard to find perfect hyperparameters, since we need the Generator to have more creativity. As a complement, we combine GAN with the HS. The Generator generates lots of samples, and some of them might be marginal samples, while the HS program will try to lead these marginal samples to perfect samples. 


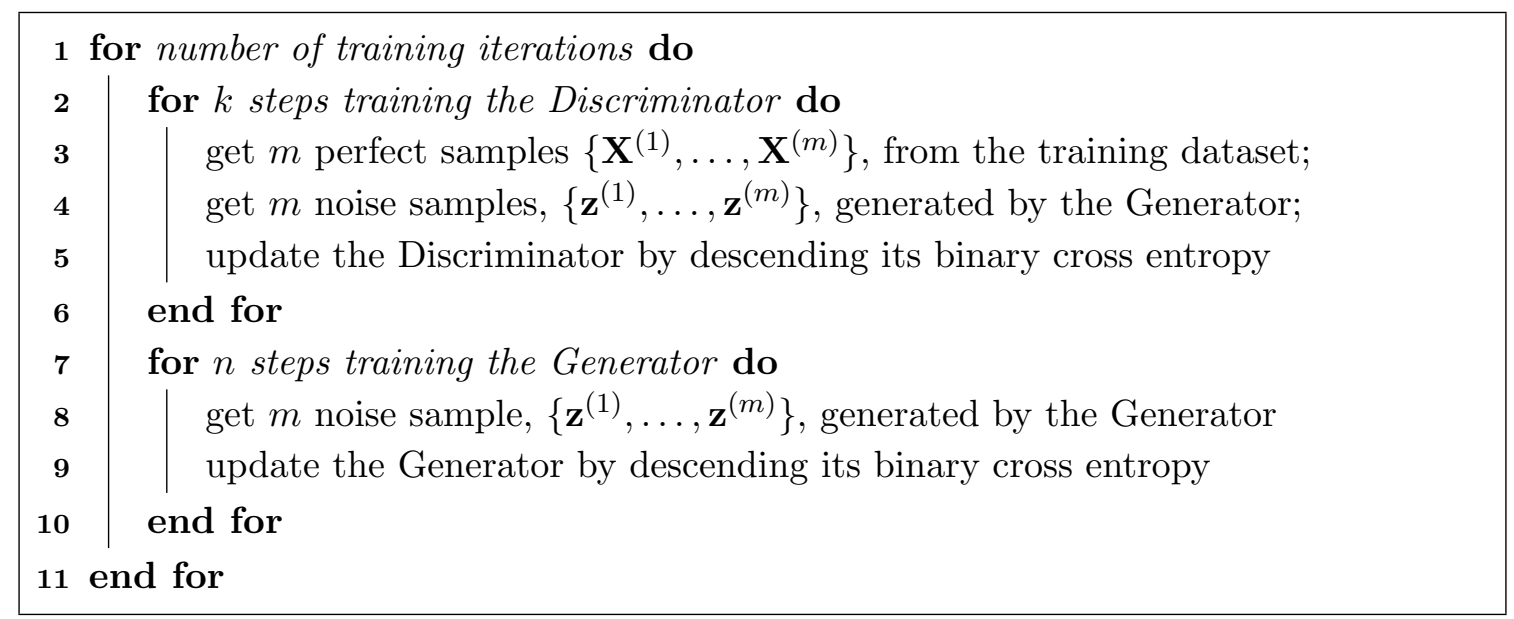

Algorithm 2. Training the Generative Adversarial Network (GAN).

\section{Results and discussions}

To satisfy all the constraints including muon g-2, dark matter, Higgs data, gluino and other SUSY search results, and try to get right dark matter relic density and large Higgs invisible decay, we consider following parameter space in the scNMSSM:

$$
\begin{aligned}
& 0.1<\mu<0.2 \mathrm{TeV}, \quad 0<M_{0}<0.5 \mathrm{TeV}, \quad 0.5<M_{1 / 2}<2 \mathrm{TeV}, \\
& 0.0<\lambda<0.7, \quad|\kappa|<0.7, \quad 1<\tan \beta<30, \\
& \left|A_{0}\right|<10 \mathrm{TeV}, \quad\left|A_{\lambda}\right|<10 \mathrm{TeV}, \quad\left|A_{\kappa}\right|<10 \mathrm{TeV} .
\end{aligned}
$$

\subsection{Scan with HS and GAN}

We developed the Heuristically Search program based on NMSSMTools-5.5.2 [50-53]. During the scan, we first require the samples satisfying the following other basic constraints:

- Theoretical constraints of vacuum stability, and without Landau pole below $M_{\mathrm{GUT}}[50-52]$.

- The lower mass bounds of charginos and sleptons from the LEP:

$$
m_{\tilde{\tau}} \geq 93.2 \mathrm{GeV}, \quad m_{\tilde{\chi}_{1}^{ \pm}} \geq 103.5 \mathrm{GeV}
$$

- Constraints from B physics, such as $B_{s} \rightarrow \mu^{+} \mu^{-}, B_{d} \rightarrow \mu^{+} \mu^{-}, b \rightarrow s \gamma$ and the mass differences $\Delta m_{d}, \Delta m_{s}[54-57]$

$$
\begin{aligned}
1.7 \times 10^{-9} & <\operatorname{Br}\left(B_{s} \rightarrow \mu^{+} \mu^{-}\right)<4.5 \times 10^{-9} \\
1.1 \times 10^{-10} & <\operatorname{Br}\left(B_{d} \rightarrow \mu^{+} \mu^{-}\right)<7.1 \times 10^{-10} \\
2.99 \times 10^{-4} & <\operatorname{Br}(b \rightarrow s \gamma)<3.87 \times 10^{-4}
\end{aligned}
$$

- An SM-liked Higgs boson exists with a mass between $123 \sim 127 \mathrm{GeV}$, and satisfies the global fit results with Higgs data at Run I and Run II of the LHC [3, 58, 59]. 


\begin{tabular}{|c|c|c|}
\hline & lower limit & upper limit \\
\hline $\begin{array}{c}\text { The DM relic } \\
\text { density } \Omega h^{2}\end{array}$ & None & 0.131 \\
\hline $\begin{array}{c}\text { The spin-independent } \\
\text { DM-nucleon cross section }\end{array}$ & None & XENON1T \\
\hline $\begin{array}{c}\text { The spin-dependent } \\
\text { DM-neutron cross section }\end{array}$ & None & LUX and XENON1T \\
\hline $\begin{array}{c}\text { The spin-dependent } \\
\text { DM-proton cross section }\end{array}$ & None & LUX, XENON1T and PICO-60 \\
\hline Muon g-2 $\delta a_{\mu}$ & $8.8 \times 10^{-10}$ & $46 \times 10^{-10}$ \\
\hline
\end{tabular}

Table 2. The upper and lower bounds of the dark matter and muon g-2 observables.

- To study Higgs invisible decay we require the mass of $\tilde{\chi}_{1}^{0}$ lighter than half of the SM-like Higgs,

$$
m_{\tilde{\chi}_{1}^{0}}<\frac{1}{2} m_{h_{\mathrm{SM}}}
$$

Then for the marginal samples, we consider the constraints of dark matter and muon g-2, calculating the score in eq. (2.26) for each sample. The upper and lower bounds of these observables are given in table 2 . The detail experimental constraints we consider in this work are list as following:

- The DM relic density $\Omega h^{2}$ from WMAP/Planck [54,60,61], we only take upper bound $\Omega h^{2} \leq 0.131$, considering there may be other sources of DM that contribute to $\Omega h^{2}$; where the dark matter observables are calculated by micrOMEGAs 5.0 [62-65] inside NMSSMTools.

- The spin-independent DM-nucleon cross section is constrained by XENON1T [66], where we rescale the original values by $\Omega / \Omega_{0}$ with $\Omega_{0} h^{2}=0.1187$;

- The spin-dependent DM-nucleon cross section is constrained by LUX [67], XENON1T [68] and PICO-60 [69], where we also rescale the original values by $\Omega / \Omega_{0}$;

- The muon anomalous magnetic moment (muon g-2) is constrained at $2 \sigma$ level including all errors. The difference between experimental result and SM theoretical value, including the corresponding error is given by [70-74]

$$
\delta a_{\mu} \equiv a_{\mu}^{\mathrm{ex}}-a_{\mu}^{\mathrm{SM}}=(27.4 \pm 9.3) \times 10^{-10}
$$

where $a_{\mu}^{\mathrm{SM}}$ contains no Higgs contribution, since we consider a SM-like Higgs in SUSY contribution to $\delta a_{\mu}$. We also consider the theoretical error of SUSY contribution, which is about $1.5 \times 10^{-10}$. Thus at $2 \sigma$ level, the central value of SUSY (including Higgses) contribution to muon g-2, $\delta a_{\mu}$, can be $5.8 \sim 49.0 \times 10^{-10}$.

If a sample satisfies the basic constraints (not including DM and muon g-2), it will get a score as a marginal or perfect sample; otherwise, it will be discarded. Then with 


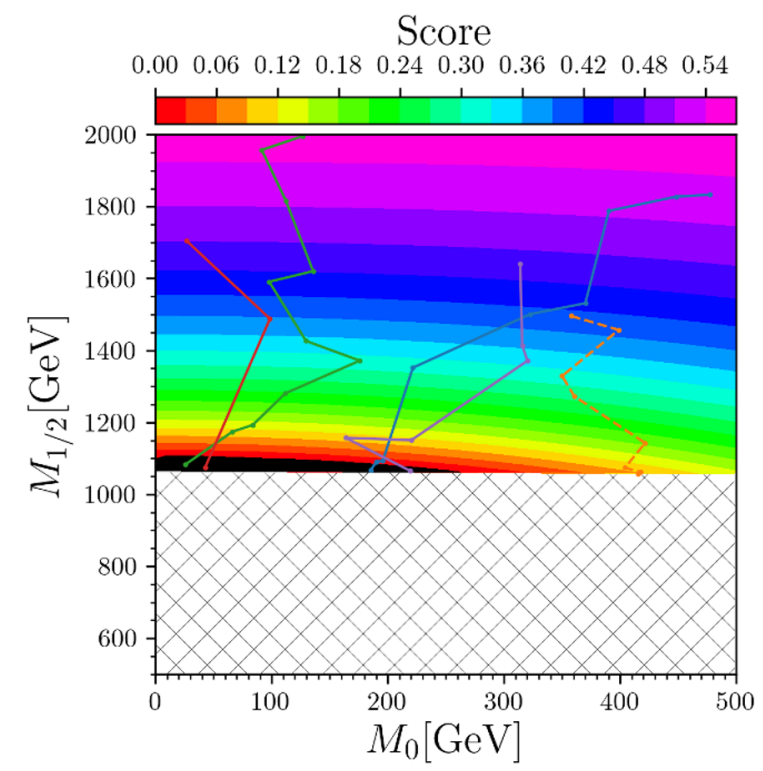

Figure 1. The samples with seven parameters fixed $(\lambda=0.278, \kappa=-0.0577, \tan \beta=17$, $\mu=162 \mathrm{GeV}, A_{0}=-1924 \mathrm{GeV}, A_{\lambda}=2756 \mathrm{GeV}$, and $\left.A_{\kappa}=589 \mathrm{GeV}\right)$ are projected in the $M_{0}$ versus $M_{1 / 2}$ plane. The colored area indicate where the samples are marginal samples, and the colors indicate their score. The black area indicate where the samples are perfect samples with zero score. While the grid area indicate this piece of parameter space is excluded by the basic constraints. The four solid lines indicate the marginal samples are led to become perfect samples, whereas the dashed line indicates that the marginal sample is failed to shift to a perfect sample.

the HS program, we did our first scan. We randomly searched for marginal samples in the parameter space, and then used the HS program changing them into perfect samples. In the first search, we got about 10k perfect samples in 24 hours. ${ }^{2}$ In fact if we changed the random scan into a multi-path Markov Chain Monte Carlo (MCMC) scan, the scan would be more efficient.

In figure 1 , we show the score of marginal samples in the $M_{0}$ versus $M_{1 / 2}$ plane. Notice that if the score equal to zero, the marginal sample is also a perfect sample. We can see that the area of marginal samples (colored range) is much larger than the perfect samples (black range) which get a zero score (satisfying all above constraints, including the DM and muon g-2). Besides we also show five tries, that the HS program tries to shift marginal samples to perfect samples, where four get success (solid lines) and one gets failure (dashed line). As the successful tries showed, the Heuristically Search usually needs less than 10 steps to shift a marginal sample to a perfect sample. In fact, many marginal samples need only several steps to change into perfect samples, while the direct search for perfect samples will waste much more time. That is the reason why we developed the HS program.

After the first search, all of the 10k perfect samples are used as the training set for the GAN. Then we trained the GAN according to algorithm 2. With a well-trained GAN, ${ }^{3}$

\footnotetext{
${ }^{2}$ We used 40 threads parallel runing on Intel(R) Xeon(R) CPU E7-4830 v3 @ 2.10GHz.

${ }^{3}$ We used Pytorch v1.3 to develop the GAN, and training cost about 5 hours. CPU: I5 6600K, GPU: GTX 1660 super.
} 

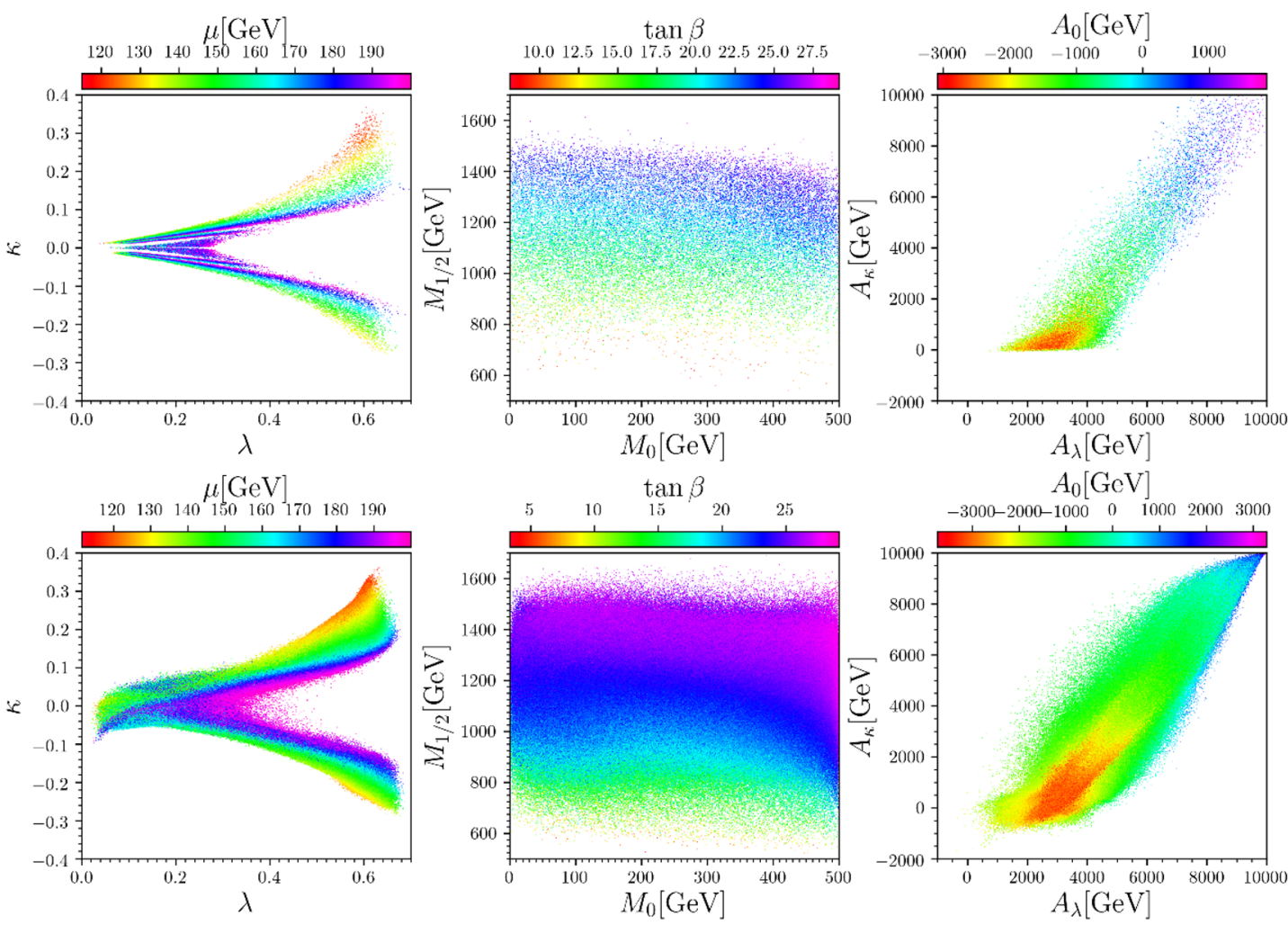

Figure 2. The samples' distributions in the $\kappa$ versus $\lambda$ (left), $M_{1 / 2}$ versus $M_{0}$ (middle), $A_{\kappa}$ versus $A_{\lambda}$ (right) planes. From left to the right, colors indicate the $\mu, \tan \beta$ and $A_{0}$, respectively. Upper panel: the training set for GAN, which are the original perfect samples generated in our first search. Lower panel: the recommended samples from GAN, which are generated by the Generator $\mathbf{G}$ in our well-trained GAN.

we can transform random noises to recommended samples that have similar distribution as the training data. Then we can easily get millions of recommended samples from the GAN in a few seconds.

In figure 2, we show the training set in the upper panels, and the recommended samples from GAN in the lower panels. We can see that the GAN has already learned the general distribution of the perfect samples in the training set. While the recommended samples from GAN (in the lower panels) have some creativity, which is not totally identical to the training set (in the upper panels). The well-trained GAN can exploit the parameter space and recommend samples around the training samples, which is exactly what we need.

We used the trained GAN to generate $2000 \mathrm{k}$ recommended samples ${ }^{4}$ and passed these recommended samples to the HS program. Then we got $280 \mathrm{k}$ perfect samples within 30 hours, ${ }^{5}$ such a way is much faster than the traditional parameter scan. At last, we impose the following additional constraints:

- The upper limit of Higgs invisible decay, 19\%, given by the CMS collaboration [7].

\footnotetext{
${ }^{4}$ Less than 1 minute on the computer with CPU: I5 6600K, GPU: GTX 1660 super.

${ }^{5}$ We used 40 threads parallel running on Intel(R) Xeon(R) CPU E7-4830 v3 @ 2.10GHz.
} 

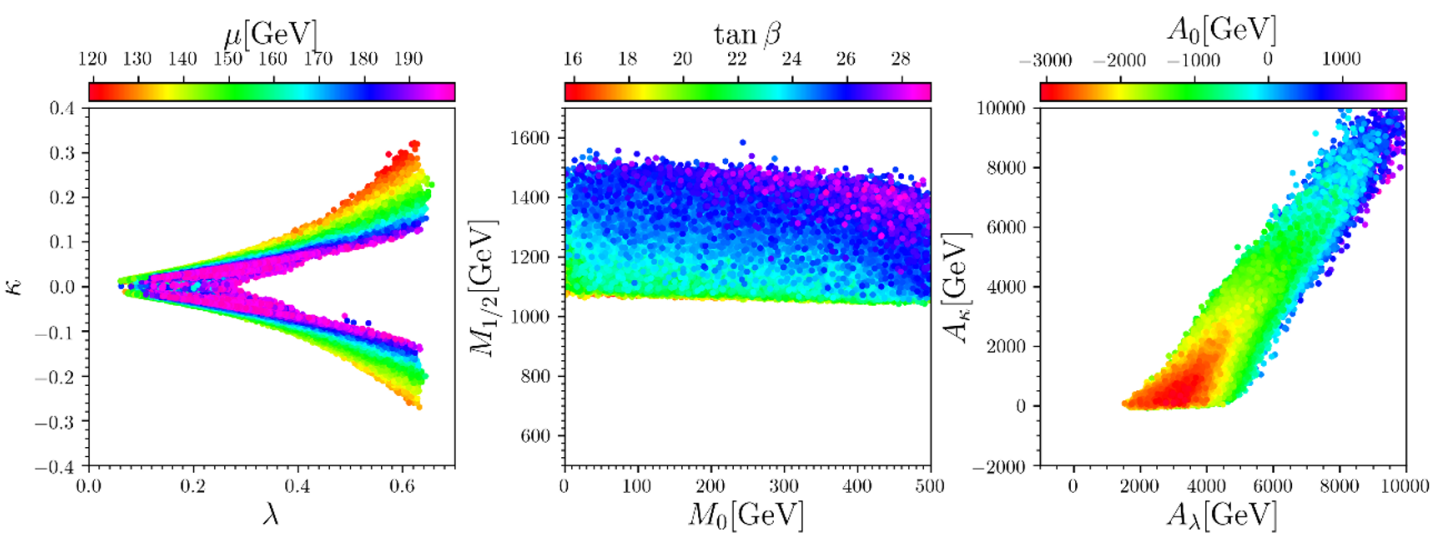

Figure 3. The final surviving samples in the $\kappa$ versus $\lambda$ (left), $M_{1 / 2}$ versus $M_{0}$ (middle), $A_{\kappa}$ versus $A_{\lambda}$ (right) planes. From left to the right, colors indicate the $\mu, \tan \beta$ and $A_{0}$, respectively.

- The lower mass bound of colored sparticles,

$$
m_{\tilde{g}}>2 \mathrm{TeV}, m_{\tilde{t}_{1}}>0.7 \mathrm{TeV}, m_{\tilde{q}_{1,2}}>2 \mathrm{TeV} .
$$

- The CMS constraints on charginos and neutralinos [75] implemented inside NMSSMTools-5.5.2.

- The SUSY search results implemented inside SModelS v1.2.2 [76-82] with official 1.2.2 database.

- The low- and high-mass resonances search results at the LEP, Tevatron and LHC, which are implemented inside HiggsBounds-5.5.0 [83-87].

Finally, after all the scans and constraints, we got about $88 \mathrm{k}$ surviving samples. In figure 3 , we show the nine free parameters of these surviving samples, and the coordinates are the same as those in figure 2 . We can see that all $M_{1 / 2}$ are larger than $1200 \mathrm{GeV}$. The reason is that we imposed the additional constraints, especially the high mass bound of gluino and the first-two-generation squarks at the LHC in eq. (3.8).

Comparing figure 3 with the lower panels in figure 2, we can see that the recommended samples from GAN are changed to perfect samples by HS program. While comparing figure 3 with the upper plane in figure 2, we can see that the GAN has recommended many marginal samples that we need, and it does have some creativity to recommend samples around the training samples. So, the combination of HS and GAN is very crucial.

\subsection{Light dark matter (DM) and Higgs invisible decay}

In figure 4 we show the final surviving samples in the plane of $\kappa$ vs $\lambda$, with colors indicate the masses of the lightest neutralino $\tilde{\chi}_{1}^{0}$, the lightest CP-even Higgs $h_{1}$ and the light CPodd Higgs $a_{1}$ respectively. For the surviving samples, we checked that the lightest CP-even Higgs $h_{1}$ are all highly singlet-dominated, and the next-to-lightest CP-even Higgs $h_{2}$ is the SM-like Higgs of $125 \mathrm{GeV}$. Since we need the SM-like Higgs have a chance decaying to 

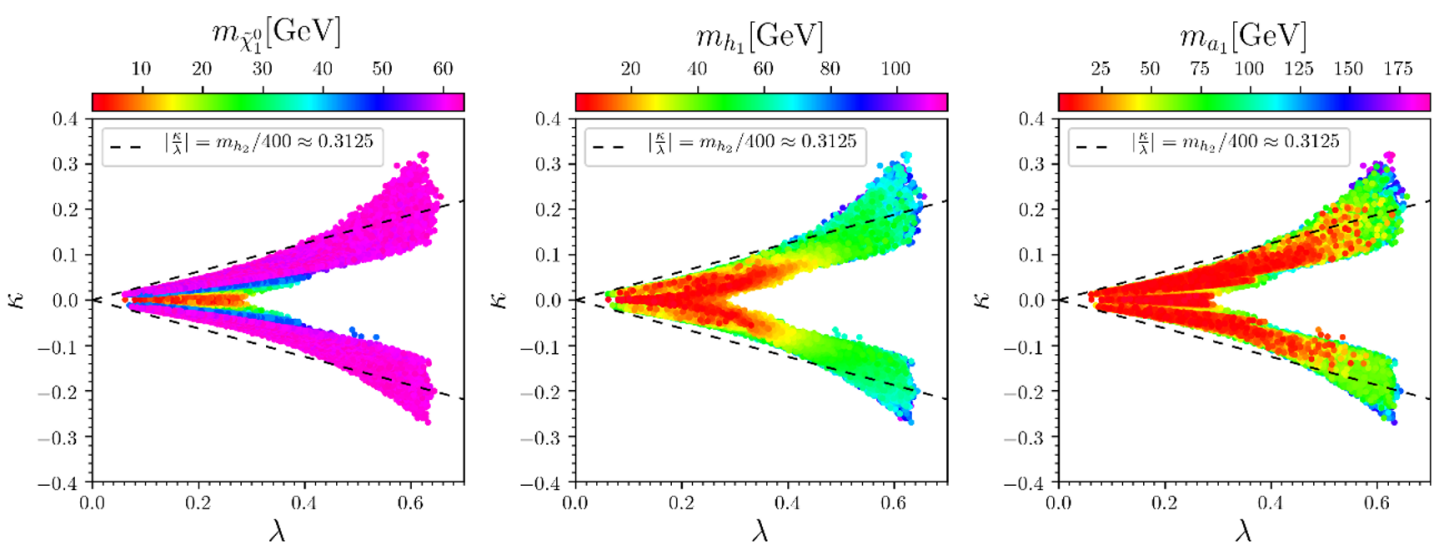

Figure 4. The final surviving samples in the $\kappa$ versus $\lambda$ planes. From left to the right, colors indicate the lightest neutralino (LSP) mass $m_{\tilde{\chi}_{1}^{0}}$, the lightest CP-even Higgs $h_{1}$ mass $m_{h_{1}}$ and the light CP-odd Higgs $a_{1}$ mass $m_{a_{1}}$, respectively. The dash line is $\left|\frac{\kappa}{\lambda}\right|=125 / 400 \approx 0.3125$. In all these planes, samples with smaller mass are projected on top of the larger ones.

invisible $\tilde{\chi}_{1}^{0}$, the $\tilde{\chi}_{1}^{0}$ is lighter than $m_{h_{2}} / 2$. If the LSP $\tilde{\chi}_{1}^{0}$ is singlino-dominated, according eq. (2.23), we should have

$$
m_{\tilde{\chi}_{1}^{0}}=2 \kappa v_{s}=2 \frac{\kappa}{\lambda} \mu \leq m_{h_{2}} / 2 .
$$

Since we set the parameter $\mu$ from 100 to $200 \mathrm{GeV}$, we have

$$
\left[\frac{\kappa}{\lambda}\right]_{\max } \leq\left[\frac{m_{h_{2}}}{4 \mu}\right]_{\min }=\frac{m_{h_{2}}}{4 \times 100} \approx 0.3125
$$

Thus it is and we checked that the $\tilde{\chi}_{1}^{0}$ are singlino-dominated for samples between the two dash line. We can also see that for the samples between the two dash lines, $h_{1}$ and $a_{1}$ are also possibly lighter than $m_{h_{2}} / 2$.

In figure 5 we show the properties of dark matter in the scNMSSM. In the lower panels, the spin-independent dark matter and nucleon scattering cross section $\sigma_{\mathrm{SI}}$ have been rescaled by a ratio of $\Omega / \Omega_{0}$, where the $\Omega_{0}$ is the right dark matter relic density with $\Omega_{0} h^{2}=0.1187$. As seen from these panels, the samples with right relic density can be divided into three cases:

- Case I: $m_{\tilde{\chi}_{1}^{0}} \simeq m_{h_{2}} / 2$

- Case II: $m_{\tilde{\chi}_{1}^{0}} \simeq m_{Z} / 2$

- Case III: $m_{\tilde{\chi}_{1}^{0}} \lesssim 12 \mathrm{GeV}$

From figure 5, we can obtain the following observations:

- From the upper left panel, the samples with right DM relic density are all with highly singlino-dominated $\tilde{\chi}_{1}^{0}$, where $\left|N_{15}\right|^{2} \gtrsim 0.9$. 

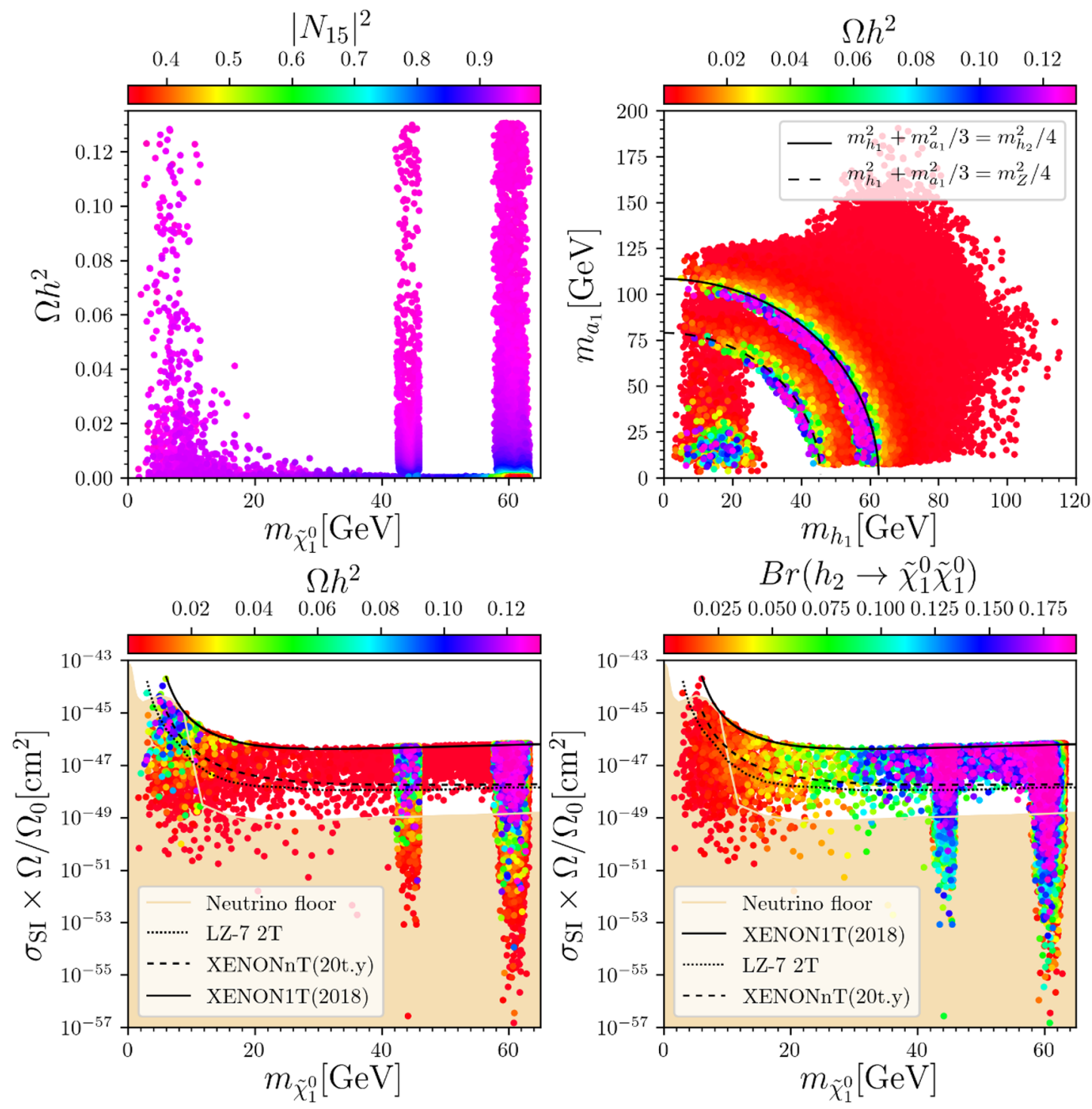

Figure 5. Upper panel: the surviving samples in the DM relic density $\Omega h^{2}$ versus the lightest neutralino (LSP) mass $m_{\tilde{\chi}_{1}^{0}}$ (left), and the CP-odd Higgs mass $m_{a_{1}}$ versus the CP-even Higgs mass $m_{h_{1}}$ (right) planes. Colors indicate the singlino component $\left|N_{15}\right|^{2}$ in the $\tilde{\chi}_{1}^{0}$ (left), and the DM relic density $\Omega h^{2}$ (right) respectively. In the right panel, the black solid and dashed curves indicate $m_{h_{1}}^{2}+m_{a_{1}}^{2} / 3=m_{Z}^{2} / 4$ and $m_{h_{1}}^{2}+m_{a_{1}}^{2} / 3=m_{h_{2}}^{2} / 4$ respectively. Samples with larger $\left|N_{15}\right|^{2}$ (left) or $\Omega h^{2}$ (right) are projected on top of the smaller ones. Lower panel: the surviving samples in the spin-independent DM-nucleon scattering cross section $\left(\sigma_{\mathrm{SI}} \times \Omega / \Omega_{0}\right)$ versus the LSP mass $m_{\tilde{\chi}_{1}^{0}}$ planes. Colors indicate the DM relic density $\Omega h^{2}$ (left), and the Higgs invisible decay $\operatorname{Br}\left(h_{2} \rightarrow \tilde{\chi}_{1}^{0} \tilde{\chi}_{1}^{0}\right)$ (right) respectively. In these two panels, the black solid, dashed and dotted curves indicate the limits of spin-independent DM-nucleon cross section $\sigma_{\mathrm{SI}}$ by XENON1T 2018 [66], the future detection sensitivity of XENONnT and LUX-ZEPLIN (LZ-7 2T), and the orange shaded region indicate the neutrino floor [88]. Samples with larger $\Omega h^{2}$ (left) or $\operatorname{Br}\left(h_{2} \rightarrow \tilde{\chi}_{1}^{0} \tilde{\chi}_{1}^{0}\right)$ (right) are projected on top of the smaller ones. 
- From the upper right panel, there is a special relationship between the mass of $h_{1}, a_{1}$ and $\tilde{\chi}_{1}^{0}$. For the samples with right DM relic density in Case I and Case II, the LSP $\tilde{\chi}_{1}^{0}$ is highly singlino-dominated, and with small $\lambda, \kappa$ and a sizable $\tan \beta$. Combining with eq. (2.25), we can see the two ellipse arcs:

$$
\begin{array}{ll}
\text { Case I : } & m_{h_{1}}^{2}+\frac{1}{3} m_{a_{1}}^{2} \simeq m_{\tilde{\chi}_{1}^{0}}^{2} \simeq\left(\frac{m_{h_{2}}}{2}\right)^{2} \\
\text { Case II : } & m_{h_{1}}^{2}+\frac{1}{3} m_{a_{1}}^{2} \simeq m_{\tilde{\chi}_{1}^{0}}^{2} \bumpeq\left(\frac{m_{Z}}{2}\right)^{2}
\end{array}
$$

- From the lower-left panel, most samples predict spin-independent DM-nucleon cross section $\sigma_{\mathrm{SI}}$ not far below the bound from XENON1T 2018, and can be covered by future LZ and XENONnT experiments. Thus these two future direct detections are crucial to check the parameter space of the scNMMSM. But there are still some samples that can escape from these future detections, and also can predict right relic density. Besides, there are also some samples below the neutrino floor, although most of them do not predict sufficient DM relic density.

- From the lower right panel, samples with large Higgs invisible decay branching ratio, $\operatorname{Br}\left(h_{2} \rightarrow \tilde{\chi}_{1}^{0} \tilde{\chi}_{1}^{0}\right)>10 \%$, have a sizable LSP mass, $m_{\tilde{\chi}_{1}^{0}}>30 \mathrm{GeV}$. This is because the small LSP mass, $m_{\tilde{\chi}_{1}^{0}}<30 \mathrm{GeV}$, always accompanying with a small $h_{1}$ and $a_{1}$ mass, which can be seen from the upper right panel of figure 4 . Then the exotic decay channels $h_{2} \rightarrow h_{1} h_{1}$ and $h_{2} \rightarrow a_{1} a_{1}$ will open, which can be seen in figure 6 . The Higgs invisible decay branching ratio $\operatorname{Br}\left(h_{2} \rightarrow \tilde{\chi}_{1}^{0} \tilde{\chi}_{1}^{0}\right)$ become smaller.

- From the lower right panel, most samples which have large Higgs invisible decay branching ratio, $\operatorname{Br}\left(h_{2} \rightarrow \tilde{\chi}_{1}^{0} \tilde{\chi}_{1}^{0}\right)>10 \%$, could be covered by future LZ and XENONnT detections. But there are still some samples that can escape from these future experiments, and also can have large Higgs invisible decay branching ratio. And there are also some samples below the neutrino floor, some of them can have large Higgs invisible decay branching ratio $\operatorname{Br}\left(h_{2} \rightarrow \tilde{\chi}_{1}^{0} \tilde{\chi}_{1}^{0}\right)>10 \%$.

In figure 6 , we show the decay information of the SM-like Higgs $h_{2}$. From this figure, we can see that all of the branching ratios of $h_{2} \rightarrow \tilde{\chi}_{1}^{0} \tilde{\chi}_{1}^{0}, h_{1} h_{1}, a_{1} a_{1}$ can be at most about $20 \%$. While we checked that considering in addition that of $h_{2}$ decay to $4 \tilde{\chi}_{1}^{0}$ though $a_{1} / h_{1} \rightarrow \tilde{\chi}_{1}^{0} \tilde{\chi}_{1}^{0}$, which acquire $m_{\tilde{\chi}_{1}^{0}}<m_{h_{2}} / 4 \simeq 31 \mathrm{GeV}$, the branching ratio of Higgs invisible decay increase very little compared with only that $h_{2}$ decay to two $\tilde{\chi}_{1}^{0}$ though $h_{2} \rightarrow \tilde{\chi}_{1}^{0} \tilde{\chi}_{1}^{0}$. The upper limit of Higgs invisible decay branching ratio is about $19 \%$ at Run II of the LHC, while the future detections for that can reach to $5.6 \%, 0.24 \%, 0.5 \%$ and $0.26 \%$ according to HL-LHC [89], CEPC [90], FCC [91] and ILC [92] respectively.

Considering the values of $\left|N_{15}\right|^{2}$, we can have the following observations from figure 6 :

- For most samples with higgsino-dominated LSP, $\left|N_{15}\right|^{2}<0.5$, the branching ratio $\operatorname{Br}\left(h_{2} \rightarrow \tilde{\chi}_{1}^{0} \tilde{\chi}_{1}^{0}\right)$ can be sizeable, while the branching ratio $\operatorname{Br}\left(h_{2} \rightarrow h_{1} h_{1}\right)$ and $\operatorname{Br}\left(h_{2} \rightarrow a_{1} a_{1}\right)$ are both zero. The reason is that the higgsino-dominated LSPs are usually accompanied by a large mass of $h_{1}$ and $a_{1}$, as can be seen from the upper panels of figure 5 , thus these two exotic decay channels are closed. 

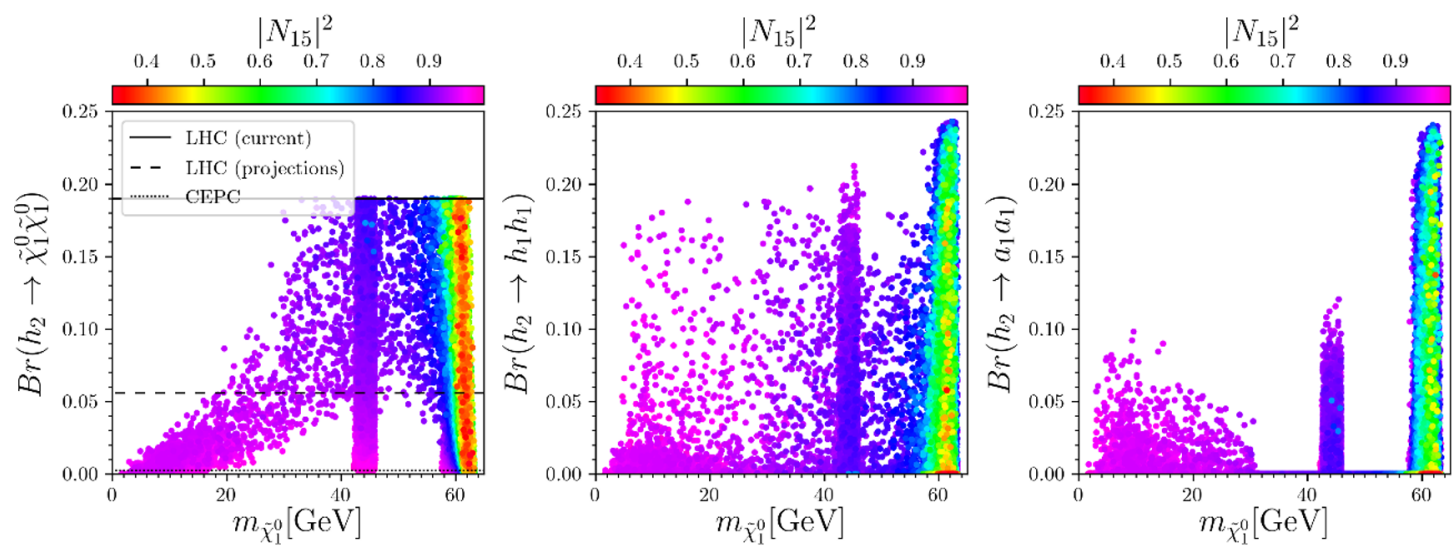

Figure 6. The surviving samples in the Higgs invisible decay $\operatorname{Br}\left(h_{2} \rightarrow \tilde{\chi}_{1}^{0} \tilde{\chi}_{1}^{0}\right)$ (left), Higgs exotic $\operatorname{Br}\left(h_{2} \rightarrow h_{1} h_{1}\right)$ (middle) and $\operatorname{Br}\left(h_{2} \rightarrow a_{1} a_{1}\right)$ (right) versus the LSP mass $m_{\tilde{\chi}_{1}^{0}}$ planes respectively. Colors indicate the singlino component $\left|N_{15}\right|^{2}$ in the LSP $\tilde{\chi}_{1}^{0}$. In the left panel, the black dashed, dash-dotted and dotted lines indicate the Higgs invisible decay upper limit from the current LHC $19 \%$ [7], future HL-LHC 5.6\% [89], and CEPC 0.24\% [90] respectively. Samples with smaller $\left|N_{15}\right|^{2}$ are projected on top of the larger ones.

- For samples with $h_{2} / Z$-funnel dark matter, $m_{\tilde{\chi}_{1}^{0}} \simeq m_{Z, h_{2}}$, the branching ratio of Higgs boson invisible decay can be large or small depending on the parameter $\lambda$.

- For most samples with low-mass LSP, $m_{\tilde{\chi}_{1}^{0}}<20 \mathrm{GeV}$, the branching ratio of Higgs boson invisible decay is small and beyond the ability of HL-LHC, while the $\operatorname{Br}\left(h_{2} \rightarrow\right.$ $\left.h_{1} h_{1}\right)$ can be larger than the $\operatorname{Br}\left(h_{2} \rightarrow a_{1} a_{1}\right)$.

- Though the detection of Higgs invisible decay, about half of the surviving samples can be covered at the future HL-LHC, while the future CEPC can cover most.

In addition, we list some discussions on other related topics in this scenario:

- We had performed a work on the annihilating mechanisms of light dark matter in this scenario [93], where we found that all the samples have the LSP in funnel mechanisms. When the LSP is lighter than $20 \mathrm{GeV}$, it is in $h_{1}$ - or $a_{1}$-funnel mechanism, that is $2 m_{\tilde{\chi}_{1}^{0}} \simeq m_{h_{1}}$ or $2 m_{\tilde{\chi}_{1}^{0}} \simeq m_{a_{1}}$.

- Higgsbounds has been used to constrain heavy Higgs bosons. We also checked that the heavy bosons $h_{3}$ and $a_{2}$ are at $2.4 \sim 4.8 \mathrm{TeV}$, and their branching ratios to $\tau$ pairs are $8 \%$ at most. The masses are not covered in ref. [94], and the production rates are much smaller than the upper limits in ref. [95]. Furthermore, we are ongoing a new work on the heavy Higgs bosons, especially on how to probe them at the future 100-TeV hadronic collider.

- We again checked the spin-dependent cross sections, and show them in figure 7. As can be seen from it, both the DM-proton and DM-neutron cross sections satisfy the current constraints. When the LSP density $\Omega h^{2}$ is sufficient, the upper limit is satisfied directly; while when the LSP density $\Omega h^{2}$ is insufficient considering there 

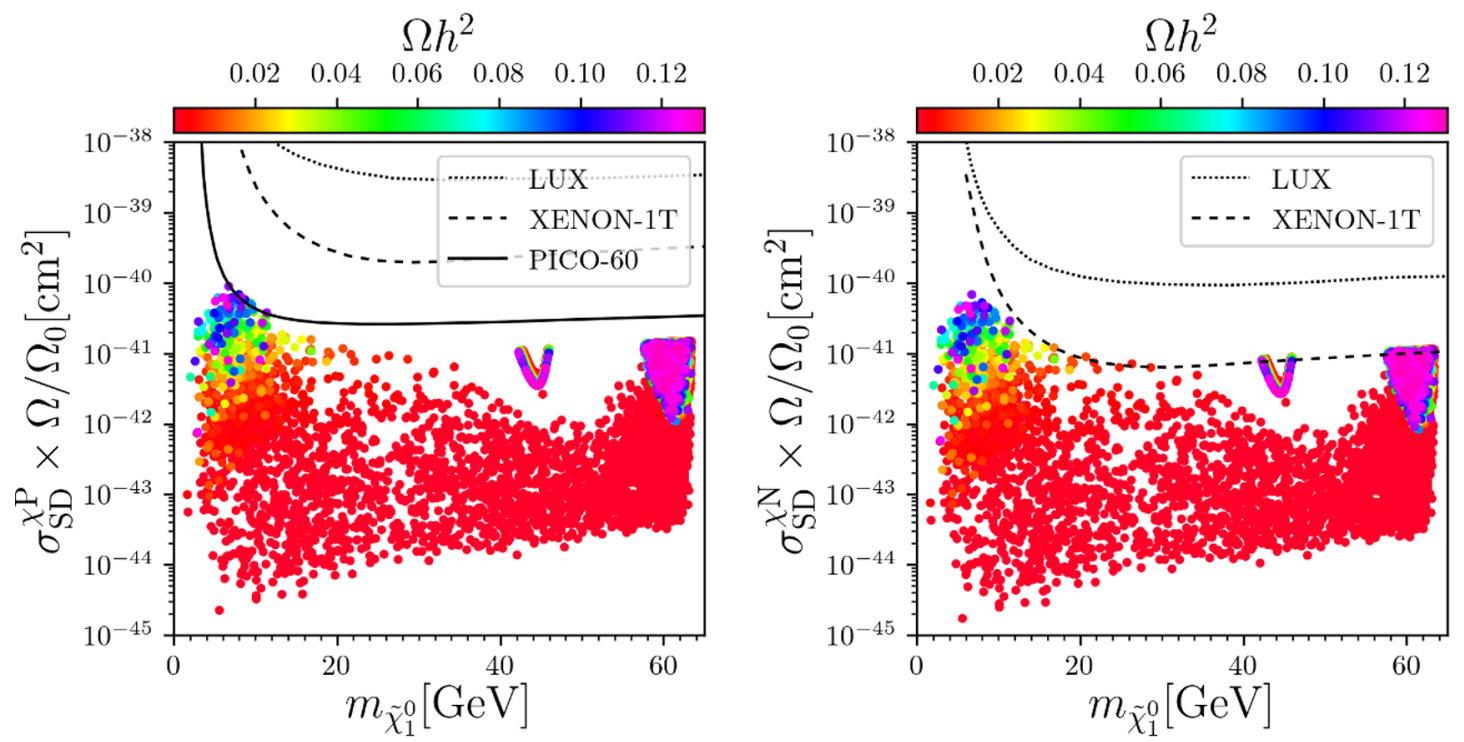

Figure 7. The surviving samples in the spin-dependent cross section of DM-proton scattering $\sigma_{\mathrm{SD}}^{\chi \mathrm{P}}$ (left) and DM-neutron scattering $\sigma_{\mathrm{SD}}^{\chi \mathrm{N}}$ (right) versus the LSP mass $m_{\tilde{\chi}_{1}^{0}}$ planes respectively. Colors indicate the LSP ratio in current dark matter $\Omega / \Omega_{0}$. In the left panel, the black dotted, dashed and solid curves indicate the limits of spin-dependent DM-proton cross section $\sigma_{\mathrm{SD}}^{\chi \mathrm{P}}$ by LUX [67], XENON-1T [68] and PICO-60 [69] respectively. While in the right panel the black dotted and dashed curves indicate these of DM-neutron cross section $\sigma_{\mathrm{SD}}^{\chi \mathrm{N}}$ by LUX [67], and XENON-1T [68] respectively. Samples with larger $\Omega h^{2}$ are projected on top of the smaller ones.

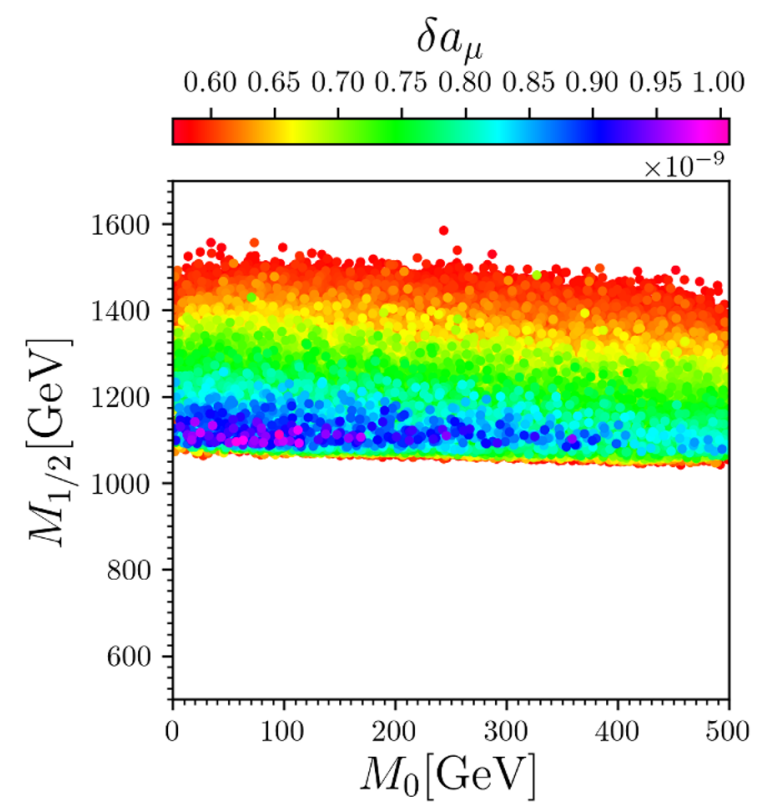

Figure 8. The surviving samples in the parameter $M_{1 / 2}$ versus $M_{0}$ planes, with colors indicating $\delta a_{\mu}$, the central value of SUSY (including Higgses) contribution to muon g-2. Samples with larger $\delta a_{\mu}$ are projected on top of the smaller ones. 
may be other sources of dark matter, the upper limit is satisfied by rescaling the cross section by a factor $\Omega / \Omega_{0}$, which is the ratio of LSP $\tilde{\chi}_{1}^{0}$ in current dark matter.

- We also checked muon g-2, and show $\delta a_{\mu}$, the central value of SUSY (including Higgses) contribution, in figure 8. When imposing the constraint, we also consider the error in SUSY-contribution calculation, which is about $1.5 \times 10^{-10}$, thus all the samples can satisfy the experimental result at $2 \sigma$ level. We also noticed that, the large $M_{1 / 2}$ values are caused by the high mass bounds of gluino and squarks in the first two generations, and this in return cause heavy wino-like chargino and bino-like neutralino, thus the SUSY contribution $\delta a_{\mu}$ cannot increase more.

\section{Conclusions}

In this work, we develop a novel scan method, combining the Heuristically Search (HS) and the Generative Adversarial Network (GAN). The HS can shift marginal samples to perfect samples, and the GAN can generate recommended samples as many as we need from noise.

In our specific process, we first scan the parameter space randomly with NMSSMTools under basic constraints, generating marginal samples; then the HS try to shift the marginal samples to perfect samples satisfying in addition the dark matter and muon g-2 constraints; with these randomly-generated perfect samples, the GAN is trained, and then generates a huge amount of recommended samples in a short time; again the HS try to shift the recommended samples to perfect samples; finally, we check the final perfect samples with additional constraints including these of sparticle searches, Higgs searches and Higgs invisible decay, getting the final surviving samples.

With this efficient method, we find a new scenario in the semi-constrained Next-to Minimal Supersymmetric Standard Model (scNMSSM), or NMSSM with non-universal Higgs masses. In this scenario,

- Both muon g-2 and right relic density can be satisfied, along with the high mass bound of gluino, etc. As far as we know, that had not been realized in the scNMSSM before this work.

- With the right relic density, the lightest neutralinos are singlino-dominated, and can be as light as $0-12 \mathrm{GeV}$.

- The future direct detections XENONnT and LUX-ZEPLIN (LZ-7 2T) can give strong constraints to this scenario.

- The current indirect constraints to Higgs invisible decay $h_{2} \rightarrow \tilde{\chi}_{1}^{0} \tilde{\chi}_{1}^{0}$ are weak, but the direct detection of Higgs invisible decay at the future HL-LHC may cover half of the samples, and that of the CEPC may cover most.

- The branching ratio of Higgs exotic decay $h_{2} \rightarrow h_{1} h_{1}, a_{1} a_{1}$ can be over 20 percent, while their contributions $\left(h_{2} \rightarrow 4 \tilde{\chi}_{1}^{0}\right)$ to the invisible decay are very small. 


\section{Acknowledgments}

This work was supported by the National Natural Science Foundation of China (NNSFC) under grant No. 11605123.

Open Access. This article is distributed under the terms of the Creative Commons Attribution License (CC-BY 4.0), which permits any use, distribution and reproduction in any medium, provided the original author(s) and source are credited.

\section{References}

[1] ATLAS collaboration, Observation of a new particle in the search for the standard model Higgs boson with the ATLAS detector at the LHC, Phys. Lett. B 716 (2012) 1 [arXiv: 1207.7214] [INSPIRE].

[2] CMS collaboration, Observation of a new boson at a mass of $125 \mathrm{GeV}$ with the CMS experiment at the LHC, Phys. Lett. B 716 (2012) 30 [arXiv:1207.7235] [INSPIRE].

[3] ATLAS collaboration, Combined measurements of Higgs boson production and decay using up to $80 \mathrm{fb}^{-1}$ of proton-proton collision data at $\sqrt{\mathrm{s}}=13 \mathrm{TeV}$ collected with the ATLAS experiment, Phys. Rev. D 101 (2020) 012002 [arXiv:1909.02845] [inSPIRE].

[4] CMS collaboration, Combined Higgs boson production and decay measurements with up to $137 \mathrm{fb}^{-1}$ of proton-proton collision data at $\sqrt{s}=13 \mathrm{TeV}$, ATLAS-CONF-2019-005 (2019).

[5] ATLAS, CMS collaboration, Precision measurements in Higgs sector at ATLAS and CMS, PoS (FFK2019) 006 [arXiv: 2001. 05927] [INSPIRE].

[6] ATLAS collaboration, Combination of searches for invisible Higgs boson decays with the ATLAS experiment, Phys. Rev. Lett. 122 (2019) 231801 [arXiv: 1904.05105] [INSPIRE].

[7] CMS collaboration, Search for invisible decays of a Higgs boson produced through vector boson fusion in proton-proton collisions at $\sqrt{s}=13$ TeV, Phys. Lett. B 793 (2019) 520 [arXiv: 1809.05937] [INSPIRE].

[8] J.-J. Cao et al., A SM-like Higgs near $125 \mathrm{GeV}$ in low energy SUSY: a comparative study for MSSM and NMSSM, JHEP 03 (2012) 086 [arXiv:1202.5821] [INSPIRE].

[9] J.-J. Cao, Z. Heng, J.M. Yang and J. Zhu, Higgs decay to dark matter in low energy SUSY: is it detectable at the LHC? JHEP 06 (2012) 145 [arXiv: 1203.0694] [INSPIRE].

[10] J. Cao, Z. Heng, J.M. Yang and J. Zhu, Status of low energy SUSY models confronted with the LHC $125 \mathrm{GeV}$ Higgs data, JHEP 10 (2012) 079 [arXiv:1207.3698] [INSPIRE].

[11] K. Kowalska et al., Constrained next-to-minimal supersymmetric standard model with a 126 GeV Higgs boson: A global analysis, Phys. Rev. D 87 (2013) 115010 [arXiv:1211.1693] [INSPIRE].

[12] J.F. Gunion, Y. Jiang and S. Kraml, The constrained NMSSM and Higgs near $125 \mathrm{GeV}$, Phys. Lett. B 710 (2012) 454 [arXiv:1201.0982] [INSPIRE].

[13] U. Ellwanger, A. Florent and D. Zerwas, Discovering the constrained NMSSM with $\tau$ leptons at the LHC, JHEP 01 (2011) 103 [arXiv:1011.0931] [INSPIRE].

[14] G. Panotopoulos, Gravitino dark matter with neutralino NLSP in the constrained NMSSM, J. Phys. Conf. Ser. 259 (2010) 012064 [arXiv:1010.4481] [InSPIRE]. 
[15] D.E. Lopez-Fogliani, L. Roszkowski, R. Ruiz de Austri and T.A. Varley, A Bayesian analysis of the constrained NMSSM, Phys. Rev. D 80 (2009) 095013 [arXiv:0906.4911] [InSPIRE].

[16] G. Bélanger, C. Hugonie and A. Pukhov, Precision measurements, dark matter direct detection and LHC Higgs searches in a constrained NMSSM, JCAP 01 (2009) 023 [arXiv:0811.3224] [INSPIRE].

[17] A. Djouadi, U. Ellwanger and A.M. Teixeira, Phenomenology of the constrained NMSSM, JHEP 04 (2009) 031 [arXiv:0811.2699] [INSPIRE].

[18] U. Ellwanger, The constrained NMSSM: mSUGRA and GMSB, AIP Conf. Proc. 1078 (2009) 73 [arXiv: 0809.0779] [INSPIRE].

[19] C. Hugonie, G. Bélanger and A. Pukhov, Dark matter in the constrained NMSSM, JCAP 11 (2007) 009 [arXiv: 0707.0628] [INSPIRE].

[20] D.G. Cerdeño et al., The constrained NMSSM with right-handed neutrinos, Eur. Phys. J. C 78 (2018) 290 [arXiv: 1707.03990] [INSPIRE].

[21] J. Cao, Z. Heng, D. Li and J.M. Yang, Current experimental constraints on the lightest Higgs boson mass in the constrained MSSM, Phys. Lett. B 710 (2012) 665 [arXiv:1112.4391] [INSPIRE].

[22] J. Ellis and K.A. Olive, Revisiting the Higgs mass and dark matter in the CMSSM, Eur. Phys. J. C 72 (2012) 2005 [arXiv: 1202.3262] [INSPIRE].

[23] P. Bechtle et al., Killing the CMSSM softly, Eur. Phys. J. C 76 (2016) 96 [arXiv: 1508.05951] [INSPIRE].

[24] GAMBIT collaboration, Global fits of GUT-scale SUSY models with GAMBIT, Eur. Phys. J. C 77 (2017) 824 [arXiv:1705.07935] [INSPIRE].

[25] K. Wang and J. Zhu, The light higgsino-dominated NLSPs in the semi-constrained NMSSM, Chin. Phys. C 44 (2020) 061001 [arXiv: 1911.08319] [inSPIRE].

[26] K. Wang, F. Wang, J. Zhu and Q. Jie, The semi-constrained NMSSM in light of muon g-2, LHC and dark matter constraints, Chin. Phys. C 42 (2018) 103109 [arXiv:1811.04435] [INSPIRE].

[27] U. Ellwanger and C. Hugonie, The semi-constrained NMSSM satisfying bounds from the LHC, LUX and Planck, JHEP 08 (2014) 046 [arXiv:1405.6647] [INSPIRE].

[28] D. Das, U. Ellwanger and A.M. Teixeira, LHC constraints on $M_{1 / 2}$ and $m_{0}$ in the semi-constrained NMSSM, JHEP 04 (2013) 117 [arXiv:1301.7584] [INSPIRE].

[29] U. Ellwanger and C. Hugonie, The higgsino-singlino sector of the NMSSM: combined constraints from dark matter and the LHC, Eur. Phys. J. C 78 (2018) 735 [arXiv: 1806.09478] [INSPIRE].

[30] U. Ellwanger, Present status and future tests of the Higgsino-singlino sector in the NMSSM, JHEP 02 (2017) 051 [arXiv: 1612.06574] [INSPIRE].

[31] K. Nakamura and D. Nomura, Charged lepton flavor violation in the semi-constrained nmssm with right-handed neutrinos, Phys. Lett. B 746 (2015) 396 [arXiv:1501.05058] [INSPIRE].

[32] J. Ren, L. Wu, J.M. Yang and J. Zhao, Exploring supersymmetry with machine learning, Nucl. Phys. B 943 (2019) 114613 [arXiv:1708.06615] [INSPIRE]. 
[33] M. Abdughani, J. Ren, L. Wu, J.M. Yang and J. Zhao, Supervised deep learning in high energy phenomenology: a mini review, Commun. Theor. Phys. 71 (2019) 955 [arXiv: 1905.06047] [INSPIRE].

[34] F. Staub, xBIT: an easy to use scanning tool with machine learning abilities, arXiv: 1906.03277 [INSPIRE].

[35] I.J. Goodfellow et al., Generative adversarial networks, arXiv:1406.2661 [INSPIRE].

[36] M. Paganini, L. de Oliveira and B. Nachman, CaloGAN: simulating 3D high energy particle showers in multilayer electromagnetic calorimeters with generative adversarial networks, Phys. Rev. D 97 (2018) 014021 [arXiv: 1712.10321] [INSPIRE].

[37] P. Musella and F. Pandolfi, Fast and accurate simulation of particle detectors using generative adversarial networks, Comput. Softw. Big Sci. 2 (2018) 8 [arXiv:1805.00850] [INSPIRE].

[38] M. Erdmann, J. Glombitza and T. Quast, Precise simulation of electromagnetic calorimeter showers using a Wasserstein Generative Adversarial Network, Comput. Softw. Big Sci. 3 (2019) 4 [arXiv: 1807.01954] [INSPIRE].

[39] R. Di Sipio, M. Faucci Giannelli, S. Ketabchi Haghighat and S. Palazzo, DijetGAN: a generative-adversarial network approach for the simulation of $Q C D$ dijet events at the LHC, JHEP 08 (2020) 110 [arXiv: 1903.02433] [INSPIRE].

[40] S. Otten et al., Event generation and statistical sampling for physics with deep generative models and a density information buffer, arXiv:1901.00875 [INSPIRE].

[41] J. Lin, W. Bhimji and B. Nachman, Machine learning templates for QCD factorization in the search for physics beyond the standard model, JHEP 05 (2019) 181 [arXiv:1903.02556] [INSPIRE].

[42] A. Butter, T. Plehn and R. Winterhalder, How to GAN LHC events, SciPost Phys. 7 (2019) 075 [arXiv: 1907.03764] [INSPIRE].

[43] M. Bellagente et al., How to GAN away detector effects, SciPost Phys. 8 (2020) 070 [arXiv: 1912.00477] [INSPIRE].

[44] A. Butter, T. Plehn and R. Winterhalder, How to GAN event subtraction, arXiv:1912.08824 [INSPIRE].

[45] D.J. Miller, R. Nevzorov and P.M. Zerwas, The Higgs sector of the next-to-minimal supersymmetric standard model, Nucl. Phys. B 681 (2004) 3 [hep-ph/0304049] [INSPIRE].

[46] M. Carena et al., Alignment limit of the NMSSM Higgs sector, Phys. Rev. D 93 (2016) 035013 [arXiv: 1510.09137] [INSPIRE].

[47] U. Ellwanger, C. Hugonie and A.M. Teixeira, The next-to-minimal supersymmetric standard model, Phys. Rept. 496 (2010) 1 [arXiv:0910.1785] [INSPIRE].

[48] D. Das, U. Ellwanger and A.M. Teixeira, Modified signals for supersymmetry in the NMSSM with a singlino-like LSP, JHEP 04 (2012) 067 [arXiv:1202.5244] [INSPIRE].

[49] M.D. Zeiler, Adadelta: an adaptive learning rate method, arXiv:1212.5701.

[50] U. Ellwanger, J.F. Gunion and C. Hugonie, NMHDECAY: a Fortran code for the Higgs masses, couplings and decay widths in the NMSSM, JHEP 02 (2005) 066 [hep-ph/0406215] [INSPIRE]. 
[51] U. Ellwanger and C. Hugonie, NMHDECAY 2.0: an updated program for sparticle masses, Higgs masses, couplings and decay widths in the NMSSM, Comput. Phys. Commun. 175 (2006) 290 [hep-ph/0508022] [INSPIRE].

[52] U. Ellwanger and C. Hugonie, NMSPEC: a Fortran code for the sparticle and Higgs masses in the NMSSM with GUT scale boundary conditions, Comput. Phys. Commun. 177 (2007) 399 [hep-ph/0612134] [INSPIRE].

[53] D. Das, U. Ellwanger and A.M. Teixeira, NMSDECAY: a Fortran code for supersymmetric particle decays in the next-to-minimal supersymmetric standard model, Comput. Phys. Commun. 183 (2012) 774 [arXiv:1106.5633] [INSPIRE].

[54] Particle Data Group collaboration, Review of particle physics, Phys. Rev. D98 (2018) 030001.

[55] LHCb collaboration, First evidence for the decay $B_{s}^{0} \rightarrow \mu^{+} \mu^{-}$, Phys. Rev. Lett. 110 (2013) 021801 [arXiv: 1211.2674] [INSPIRE].

[56] BABAR collaboration, Evidence for an excess of $\bar{B} \rightarrow D^{(*)} \tau^{-} \bar{\nu}_{\tau}$ decays, Phys. Rev. Lett. 109 (2012) 101802 [arXiv: 1205.5442] [INSPIRE].

[57] BABAR collaboration, Precision measurement of the $B \rightarrow X_{s} \gamma$ photon energy spectrum, branching fraction and direct $C P$ asymmetry $A_{C P}\left(B \rightarrow X_{s+d} \gamma\right)$, Phys. Rev. Lett. 109 (2012) 191801 [arXiv: 1207.2690] [INSPIRE].

[58] CMS collaboration, Combined measurements of Higgs boson couplings in proton-proton collisions at $\sqrt{s}=13 \mathrm{TeV}$, Eur. Phys. J. C 79 (2019) 421 [arXiv:1809.10733] [INSPIRE].

[59] ATLAS, CMS collaboration, Measurements of the Higgs boson production and decay rates and constraints on its couplings from a combined ATLAS and CMS analysis of the LHC pp collision data at $\sqrt{s}=7$ and $8 \mathrm{TeV}$, JHEP 08 (2016) 045 [arXiv: 1606.02266] [INSPIRE].

[60] WMAP collaboration, Nine-Year Wilkinson Microwave Anisotropy Probe (WMAP) observations: cosmological parameter results, Astrophys. J. Suppl. 208 (2013) 19 [arXiv:1212.5226] [INSPIRE].

[61] Planck collaboration, Planck 2013 results. XVI. Cosmological parameters, Astron. Astrophys. 571 (2014) A16 [arXiv:1303.5076] [INSPIRE].

[62] G. Bélanger, F. Boudjema, A. Pukhov and A. Semenov, MicrOMEGAs 2.0: a program to calculate the relic density of dark matter in a generic model, Comput. Phys. Commun. 176 (2007) 367 [hep-ph/0607059] [INSPIRE].

[63] G. Bélanger, F. Boudjema, A. Pukhov and A. Semenov, Dark matter direct detection rate in a generic model with MicrOMEGAs 2.2, Comput. Phys. Commun. 180 (2009) 747 [arXiv:0803.2360] [INSPIRE].

[64] G. Bélanger, F. Boudjema, A. Pukhov and A. Semenov, MicrOMEGAs: a tool for dark matter studies, Nuovo Cim. C 033N2 (2010) 111 [arXiv:1005.4133] [INSPIRE].

[65] G. Bélanger, F. Boudjema, A. Pukhov and A. Semenov, MicrOMEGAs_3: a program for calculating dark matter observables, Comput. Phys. Commun. 185 (2014) 960 [arXiv: 1305.0237] [INSPIRE].

[66] XENON collaboration, Dark matter search results from a one ton-year exposure of XENON1T, Phys. Rev. Lett. 121 (2018) 111302 [arXiv:1805.12562] [INSPIRE]. 
[67] LUX collaboration, Results on the spin-dependent scattering of weakly interacting massive particles on nucleons from the Run 3 data of the LUX experiment, Phys. Rev. Lett. 116 (2016) 161302 [arXiv:1602.03489] [INSPIRE].

[68] XENON collaboration, Constraining the spin-dependent WIMP-nucleon cross sections with XENON1T, Phys. Rev. Lett. 122 (2019) 141301 [arXiv:1902.03234] [inSPIRE].

[69] PICO collaboration, Dark matter search results from the complete exposure of the PICO-60 $C_{3} F_{8}$ bubble chamber, Phys. Rev. D 100 (2019) 022001 [arXiv:1902.04031] [INSPIRE].

[70] Muon G-2 collaboration, Final Report of the Muon E821 Anomalous Magnetic Moment Measurement at BNL, Phys. Rev. D 73 (2006) 072003 [hep-ex/0602035] [INSPIRE].

[71] A. Czarnecki, W.J. Marciano and A. Vainshtein, Refinements in electroweak contributions to the muon anomalous magnetic moment, Phys. Rev. D 67 (2003) 073006 [Erratum ibid. D 73 (2006) 119901] [hep-ph/0212229] [INSPIRE].

[72] S. Heinemeyer, D. Stöckinger and G. Weiglein, Electroweak and supersymmetric two-loop corrections to $(g-2)_{\mu}$, Nucl. Phys. B 699 (2004) 103 [hep-ph/0405255] [INSPIRE].

[73] J. Bijnens and J. Prades, The hadronic light-by-light contribution to the muon anomalous magnetic moment: where do we stand?, Mod. Phys. Lett. A 22 (2007) 767 [hep-ph/0702170] [INSPIRE].

[74] F. Jegerlehner, Essentials of the muon g-2, Acta Phys. Polon. B 38 (2007) 3021 [hep-ph/0703125] [INSPIRE].

[75] CMS collaboration, Combined search for electroweak production of charginos and neutralinos in proton-proton collisions at $\sqrt{s}=13 \mathrm{TeV}$, JHEP 03 (2018) 160 [arXiv:1801.03957] [INSPIRE].

[76] S. Kraml et al., SModelS: a tool for interpreting simplified-model results from the LHC and its application to supersymmetry, Eur. Phys. J. C 74 (2014) 2868 [arXiv:1312.4175] [INSPIRE].

[77] F. Ambrogi et al., SModelS v1.1 user manual: improving simplified model constraints with efficiency maps, Comput. Phys. Commun. 227 (2018) 72 [arXiv:1701.06586] [INSPIRE].

[78] F. Ambrogi et al., SModelS v1.2: long-lived particles, combination of signal regions and other novelties, Comput. Phys. Commun. 251 (2020) 106848 [arXiv:1811.10624] [INSPIRE].

[79] J. Dutta, S. Kraml, A. Lessa and W. Waltenberger, SModelS extension with the CMS supersymmetry search results from Run 2, LHEP 1 (2018) 5 [arXiv: 1803. 02204] [INSPIRE].

[80] A. Buckley, PySLHA: a Pythonic interface to SUSY Les Houches Accord data, Eur. Phys. J. C 75 (2015) 467 [arXiv: 1305.4194] [INSPIRE].

[81] T. Sjöstrand, S. Mrenna and P.Z. Skands, PYTHIA 6.4 physics and manual, JHEP 05 (2006) 026 [hep-ph/0603175] [INSPIRE].

[82] T. Sjöstrand et al., An introduction to PYTHIA 8.2, Comput. Phys. Commun. 191 (2015) 159 [arXiv: 1410.3012] [INSPIRE].

[83] P. Bechtle et al., Applying exclusion likelihoods from LHC searches to extended Higgs sectors, Eur. Phys. J. C 75 (2015) 421 [arXiv:1507.06706] [INSPIRE].

[84] P. Bechtle et al., HiggsBounds-4: improved tests of extended Higgs sectors against exclusion bounds from LEP, the Tevatron and the LHC, Eur. Phys. J. C 74 (2014) 2693 [arXiv:1311.0055] [INSPIRE]. 
[85] P. Bechtle et al., Recent developments in HiggsBounds and a preview of HiggsSignals, PoS (CHARGED2012) 024 [arXiv: 1301.2345] [INSPIRE].

[86] P. Bechtle et al., HiggsBounds 2.0.0: confronting neutral and charged Higgs sector predictions with exclusion bounds from LEP and the Tevatron, Comput. Phys. Commun. 182 (2011) 2605 [arXiv: 1102.1898] [INSPIRE].

[87] P. Bechtle et al., HiggsBounds: confronting arbitrary Higgs sectors with exclusion bounds from LEP and the Tevatron, Comput. Phys. Commun. 181 (2010) 138 [arXiv:0811.4169] [INSPIRE].

[88] J. Billard, L. Strigari and E. Figueroa-Feliciano, Implication of neutrino backgrounds on the reach of next generation dark matter direct detection experiments, Phys. Rev. D 89 (2014) 023524 [arXiv: 1307.5458 ] [INSPIRE].

[89] Z. Liu, L.-T. Wang and H. Zhang, Exotic decays of the $125 \mathrm{GeV}$ Higgs boson at future $e^{+} e^{-}$ lepton colliders, Chin. Phys. C 41 (2017) 063102 [arXiv:1612.09284] [INSPIRE].

[90] Y. Tan et al., Search for invisible decay of a Higgs boson produced at the CEPC, arXiv:2001.05912 [INSPIRE].

[91] D. d'Enterria, Higgs physics at the Future Circular Collider, PoS (ICHEP2016) 434 [arXiv: 1701.02663] [INSPIRE].

[92] A. Ishikawa, Search for invisible decays of the Higgs boson at the ILC, PoS (LeptonPhoton2019) 147 [arXiv: 1909. 07537] [INSPIRE].

[93] K. Wang and J. Zhu, Funnel annihilations of light dark matter and the invisible decay of the Higgs boson, Phys. Rev. D 101 (2020) 095028 [arXiv:2003.01662] [INSPIRE].

[94] ATLAS collaboration, Search for additional heavy neutral Higgs and gauge bosons in the ditau final state produced in $36 \mathrm{fb}^{-x 1}$ of pp collisions at $\sqrt{s}=13$ TeV with the ATLAS detector, JHEP 01 (2018) 055 [arXiv: 1709.07242] [INSPIRE].

[95] CMS collaboration, Search for additional neutral MSSM Higgs bosons in the $\tau \tau$ final state in proton-proton collisions at $\sqrt{s}=13 \mathrm{TeV}, \mathrm{JHEP} 09$ (2018) 007 [arXiv:1803.06553] [INSPIRE]. 